\title{
Cooperative success in epithelial public goods games
}

\author{
Jessie Renton*, Karen M. Page \\ Department of Mathematics, University College London, Gower Street, London WC1E 6BT, UK
}

\section{A R T I C L E I N F O}

\section{Article history:}

Received 29 January 2021

Revised 6 July 2021

Accepted 19 July 2021

Available online 22 July 2021

\section{Keywords:}

Multiplayer games

Cooperation

Evolutionary game theory

Voronoi tessellation

Epithelial automata

\begin{abstract}
A B S T R A C T
Cancer cells obtain mutations which rely on the production of diffusible growth factors to confer a fitness benefit. These mutations can be considered cooperative, and studied as public goods games within the framework of evolutionary game theory. The population structure, benefit function and update rule all influence the evolutionary success of cooperators. We model the evolution of cooperation in epithelial cells using the Voronoi tessellation model. Unlike traditional evolutionary graph theory, this allows us to implement global updating, for which birth and death events are spatially decoupled. We compare, for a sigmoid benefit function, the conditions for cooperation to be favoured and/or beneficial for wellmixed and structured populations. We find that when population structure is combined with global updating, cooperation is more successful than if there were local updating or the population were well-mixed. Interestingly, the qualitative behaviour for the well-mixed population and the Voronoi tessellation model is remarkably similar, but the latter case requires significantly lower incentives to ensure cooperation.
\end{abstract}

(C) 2021 The Author(s). Published by Elsevier Ltd. This is an open access article under the CC BY license (http://creativecommons.org/licenses/by/4.0/).

\section{Introduction}

\subsection{Cooperation between cancer cells}

Oncogenesis is a process of somatic evolution. In order to become cancerous there are certain key mutations which cells must obtain, corresponding to the hallmarks of cancer (Hanahan and Weinberg, 2000; Hanahan and Weinberg, 2011). Evolutionary game theory provides a framework for modelling mutations which have a fitness effect beyond the cell itself. For example, certain mutations can be considered cooperative, in that they invoke a cost to the cell which is recuperated as a shared benefit. This is evident when the benefit relies on the production of a diffusible growth factor (Jouanneau et al., 1994; Axelrod et al., 2006), as is the case for a number of the hallmarks of cancer, such as self-sufficiency in growth signalling and sustained angiogenesis. The Warburg effect, whereby tumour cells metabolise through glycolysis even when oxygen is abundant (Warburg, 1956), can also be considered cooperative (Archetti, 2014).

Cooperative mutations benefit the population as a whole; however, it is often the case that defection (e.g. not producing growth factor) results in higher individual fitness. This is because the defector shares in the benefits without paying any fitness costs associated with cooperating. Understanding the conditions under which cooperation can evolve, despite the incentive to defect,

\footnotetext{
* Corresponding author.
}

has been a topic of extensive study within evolutionary game theory (Nowak, 2006; Ohtsuki et al., 2006a; Allen et al., 2017).

Cooperation is usually considered to be a desirable outcome. For example, within the social sphere or amongst healthy constituent cells of a multicellular organism. Cooperation between cancer cells, however, can drive tumour growth (Marusyk etal., 2014). This is of course detrimental to the patient, and thus, disrupting cooperation between cancer subclones, possibly by exploiting its evolutionary weaknesses, could be an important avenue for treatment (Archetti, 2013a; Zhou et al., 2017).

\subsection{Public goods games}

Applications of evolutionary game theory to model cancer evolution have mainly focussed on two-player games, whereby cells participate in multiple pairwise interactions within the population (Tomlinson, 1997; Basanta and Deutsch, 2008; Hummert et al., 2014). Interactions between cancer cells, however, tend to happen in groups. For example, a cell producing a growth factor will provide a benefit to other cells within its diffusion range. These types of mutations are thus better represented as multiplayer public goods games (PGGs) (Archetti and Scheuring, 2012), played between producer (cooperator) and non-producer (defector) cells. The former produce growth factor at a fixed cost to their fitness. Both producers and non-producers receive a fitness benefit as a function of the frequency of producers in their interaction neighbourhood. 
The most common PGG, known as the N-person prisoner's dilemma (NPD), uses a linear benefit function (Hauert et al., 2002; Santos et al., 2008). However, non-linear benefit functions may be more realistic (Archetti et al., 2015; Archetti et al., 2017), and can lead to much richer dynamics, even for well-mixed populations. An example is the volunteer's dilemma (VD), which defines the benefit as a Heaviside step function (Bach et al., 2001; Bach et al., 2006; Archetti, 2009a; Archetti, 2009b).

A sigmoid benefit function has been proposed as an appropriate model for growth factor production. Experiments on neuroendocrine pancreatic cancer cells in vitro have found sigmoid dependence of proliferation rates on the concentration of growth factor IGF-II (Archetti et al., 2015). Furthermore, such a function is relatively general, with both the NPD and VD arising as extreme cases (Archetti and Scheuring, 2011).

\subsection{Population structure and update rules}

Most cancers originate in epithelia. These are tissues formed of sheets of cells, which are approximately polygonal on their apical surfaces. It is important to take into account this population structure when modelling the evolutionary dynamics. For both twoplayer cooperation games (Ohtsuki et al., 2006b; Nowak et al., 2010) and multiplayer PGGs (Peña et al., 2016), cooperators tend to have greater success in structured populations, as compared to well-mixed ones, because they are able to form mutually beneficial clusters.

Evolution on structured populations is usually modelled within the framework of evolutionary graph theory (Lieberman et al., 2005), in which the population is represented as a fixed graph. Epithelial cells tend to have six neighbours on average, and thus can be represented as a hexagonal lattice. Introducing more realistic population structures, with small variation in neighbour number, does not have a significant impact on evolutionary outcomes (Archetti, 2016; Renton and Page, 2019).

The success of cooperation is also dependent on the update dynamics. Within evolutionary graph theory, the population evolves according to an update rule. In general, update rules can be divided into two categories: local and global (Nathanson et al., 2009).

\subsubsection{Local updating}

A local update involves a spatial relationship between birth and death events. Evolutionary graph theory usually requires a local update rule in order to maintain the fixed graph structure. Two commonly used local update rules are defined as follows:

- birth-death: a cell is selected to divide with probability proportional to fitness; one of its neighbours is chosen to die uniformly at random.

- death-birth: a cell is chosen to die uniformly at random; one of its neighbours is selected to divide with probability proportional to fitness

In both cases the offspring of the dividing cell occupies the empty site left by the dead cell (Zukewich et al., 2013). The choice between these update rules has a substantive effect on evolutionary outcomes. For example, consider a two-player prisoner's dilemma game and a population represented by a regular graph. Cooperation can be favoured for a death-birth update rule, so long as the benefit is high enough. For the birth-death update, however, as is the case with a well-mixed population, cooperation is only favoured for an infinitely large benefit (Ohtsuki et al., 2006b).

These update rules are sometimes referred to as BD-B (birthdeath with selection on birth) and DB-B (death-birth with selection on birth) to emphasise that selection is acting on birth. Alternative update rules, for which selection acts on death, can then be referred to as BD-D and DB-D (Masuda, 2009). In this paper, we limit ourselves to the case where selection acts on birth, thus we do not use this notation to differentiate the two cases.

\subsubsection{Global updating}

Under a global update rule there is no spatial dependence between birth and death events; thus, cells are selected to reproduce and die from the population as a whole. Global updating is generally seen for well-mixed populations, or when populations are organised in phenotype space (Antal et al., 2009) or by sets (Tarnita et al., 2009b).

Within evolutionary graph theory the shift update rule is an example of global updating. In this case a cell is chosen to divide with probability proportional to fitness, and a second cell is chosen to die uniformly at random. A path is then selected on the graph which connects the two. Cells are shifted along this path until there is an empty node next to the dividing cell for its progeny to occupy. This kind of update works well on a one-dimensional lattice (Allen and Nowak, 2012), and promotes cooperation, even compared to the death-birth update. However, it becomes more complex in two-dimensions (Pavlogiannis et al., 2015), because division causes cellular rearrangement at a distance from the event.

\subsubsection{Epithelial structure and dynamics}

Evolutionary graph theory has several shortcomings for modelling invasion processes in epithelia. Firstly, it assumes that the population can be represented by a static graph, whereas epithelia are dynamic structures. Secondly, as we have discussed, introducing global update rules into evolutionary graph theory presents challenges to the modelling framework (Pavlogiannis et al., 2015).

The question then arises as to which update rule is most realistic for an epithelium. This will depend on the extent to which death and division processes are spatially coupled. For homeostatic tissues it is likely that contact inhibition, the phenomenon whereby cells stop proliferating at high density, plays an important role in maintaining the population size (Mesa et al., 2018). The deathbirth update rule could be an appropriate model when contact inhibition is very strong, as tissue density is likely to be low near a recent death. Conversely, a global update rule is likely to be more realistic when contact inhibition is weaker and thus there is less spatial dependence between death and division.

The death-birth and decoupled update rules represent extreme cases of spatial coupling between division and death. In this paper we focus on global updating, as the death-birth update rule, along with other local update rules, has been extensively studied within evolutionary graph theory (Ohtsuki et al., 2006b; Maciejewski et al., 2014; Peña et al., 2016; Allen et al., 2017). In future work, we will consider the spectrum of spatial coupling that can arise in a tissue due to contact inhibition, and how this affects the evolution of cooperation.

In line with our previous work (Renton and Page, 2019), we use the Voronoi tessellation (VT) model (Meineke et al., 2001; Van Leeuwen et al., 2009) to represent epithelial dynamics. Unlike traditional evolutionary graph theory models, the tissue structure is dynamic and cells are able to divide and die independently. It is thus straightforward to spatially decouple birth and death, and we are able to introduce a global form of updating, we call the decoupled update rule. In Renton and Page (2019), we used this framework to analyse the two-player prisoner's dilemma game, finding that cooperation was more successful for the decoupled update rule, than for a death-birth update rule. The present paper extends these results to a wide range of multiplayer public goods games, as well as deriving general results for global update rules.

We aim to extend the range of applicability of quasi-analytical methods from evolutionary game theory to more realistic tissue models. We have chosen to use the VT model, because it uses a very 
simple force law and, as a cell-centre model, naturally provides the graph structure needed for evolutionary games (Meineke et al., 2001). Furthermore, unlike cellular automata models, cell division leads only to local topological changes. The VT model has been used to represent cellular dynamics in colonic and intestinal crypts, including for models of invasion (Mirams et al., 2012; Romijn et al., 2020). Other tissue models, such as the vertex model (Farhadifar et al., 2007), could also be appropriate for our purposes.

The version of the VT model we use represents a simple epithelium $^{1}$ as a two-dimensional structure. Thus our results are mostly relevant to the early stages of tumorigenesis or field cancerization (Curtius et al., 2017) in simple epithelia. While models of later stage tumour evolution would be more appropriately modelled in three dimensions, two-dimensional models can still be useful in the first instance.

\subsection{Measures of mutant success}

For stochastic evolutionary games without mutation, we can compare the success of different strategies by calculating fixation probabilities. Here we consider the dynamics of two cell types: $A$ and $B$. The fixation probability $\rho_{X}$ is then defined as the probability that a single initial mutant $X$ will eventually take over the entire population. We consider two measures for the success of an $A$ mutant (Zukewich et al., 2013; Maciejewski et al., 2014):

- $A$ is a beneficial mutation when $\rho_{A}>\rho_{0}$. Here $\rho_{0}=1 / Z$ is the fixation probability for a neutral mutant and $Z$ is the population size.

- A is favoured by selection, or has an evolutionary advantage, when $\rho_{A}>\rho_{B}$. This is equivalent to the condition that the equilibrium frequency of $A$ is greater than a half when mutation is allowed ( $A$ is the dominant strategy).

In general, these conditions are not equivalent, thus it is possible for a mutation to be beneficial but not favoured, or vice versa. One or the other condition might be more relevant to quantifying mutant success depending on the circumstances. Furthermore, under certain circumstances these two conditions are equivalent (Maciejewski et al., 2014).

The remainder of this paper explores conditions under which a mutation is beneficial and/or favoured. We begin in Section 2 by setting out the mathematical formalism for multiplayer evolutionary games, focussing particularly on PGGs played between cooperators and defectors. Section 2.1 then introduces the $\sigma$-rule, which is used to determine whether a strategy is favoured. We outline several known results on graphs with local update rules, as well as deriving results for a birth-death and shift update rule on a cycle. We then derive the conditions for favourability on a general population structure with global updating. In Section 2.2 we derive a similar rule, but for a strategy to be beneficial. In Section 3 we apply this theory to consider conditions for cooperator success in an epithelium, using spatial statistics calculated through simulation of the Voronoi tessellation model. Finally, in Section 4, we discuss the implications of our work for the evolution of cooperative public goods in epithelia and make some remarks on the different significance of beneficial and favourable mutants.

\section{Evolutionary dynamics of multiplayer games}

We consider an arbitrary multiplayer game with two strategies, $A$ and $B$. Players interact in groups of size $N=k+1$, and obtain

\footnotetext{
${ }^{1}$ A simple epithelium is formed of a single layer of cells, whereas a stratified epithelium is multilayered.
}

payoffs $a_{j, k}$ and $b_{j, k}$ respectively, where $j$ is the number of $A$ coplayers and $k$ is the total number of co-players. For a graphstructured population, the co-players are direct neighbours. The fitness of each individual is then defined as $1+\delta a_{j, k}$ or $1+\delta b_{j, k}$, where $\delta$ is the selection strength parameter.

The population evolves according to a Moran process (Moran, 1958), i.e. at each time-step one individual dies and another reproduces. Thus, the population size $Z$ remains constant. How these individuals are chosen is determined by the update rule. We consider cases where reproduction, but not death, is dependent on fitness.

Many of the results we derive in the following sections are for general games, however we are focussed on PGGs played between producer/cooperator cells $(C)$ and non-producer/defector cells $(D)$. These games are defined by a benefit function $b \cdot \beta(x)$ and a cost function, which we take to be constant $c$, with $b>c$. Here, $x$ is the proportion of cooperators in a cell's interaction group. Thus, cooperator and defector payoffs are defined respectively as

$a_{j, k}=b \cdot \beta\left(\frac{j+1}{k+1}\right)-c, \quad b_{j, k}=b \cdot \beta\left(\frac{j}{k+1}\right)$.

In order to ensure that the payoff is higher when all players cooperate than when no players cooperate we enforce the condition $b \cdot \beta(1)-c \geqslant b \cdot \beta(0)$. Often this is done by setting $c=1, b>1, \beta(1)=1$ and $\beta(0)=0$.

The NPD and VD can both be defined in this form by specifying the benefit functions:

$\beta(x)=x \quad(\mathrm{NPD})$

$\beta(x)=\Theta(x-\tilde{x})$,

where $\Theta(x)$ is the Heaviside step function and $\tilde{x}$ is the minimum proportion of cooperators required to obtain the benefit. Furthermore we can define a general sigmoid benefit function:

$\beta(x)=\frac{\alpha(x)-\alpha(0)}{\alpha(1)-\alpha(0)}$,

where

$\alpha(x)=\frac{1}{1+e^{s(h-x)}}$

is the logistic function, $s$ is the steepness and $h$ is the inflection point. We can regain the NPD and VD by taking the limits $s \rightarrow 0$ and $s \rightarrow \infty$ respectively (see Fig. 1 ).

\subsection{The $\sigma$-rule: conditions for cooperation to be favoured}

For a particular update rule and population structure, the $\sigma$-rule allows us to determine which is the favoured strategy (Wu et al.,
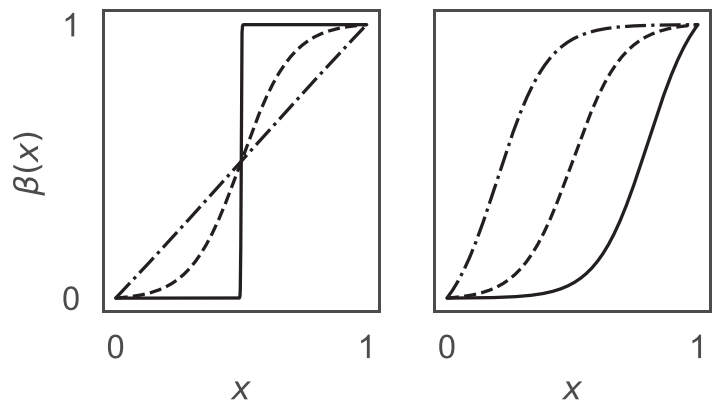

Fig. 1. Logistic benefit function. Left panel: $h=0.5 ; s=1$ (dash-dot), $s=10$ (dash) and $s=1000$ (solid). We can regain the limiting cases by letting $s \rightarrow 0$ (NPD) or $s \rightarrow \infty$ (VD). Right panel: $s=10 ; h=0.2$ (dash-dot), $h=0.5$ (dash), $h=0.8$ (solid). 
2013). We recall from Section 1.4, that a strategy $A$ is favoured over

$B$, when $\rho_{A}>\rho_{B}$.

The $\sigma$-rule states that

$\rho_{A}>\rho_{B} \Longleftrightarrow \sum_{j=0}^{k} \sigma_{j}\left(a_{j}-b_{k-j}\right)>0$,

where $\sigma_{j}$ are the structure coefficients. It is assumed that the group size $N=k+1$ is fixed. Thus, we have let $a_{j, k}=a_{j}$ and $b_{j, k}=b_{j}$. The structure coefficients are dependent on the population structure and update rule, but not the payoffs. Therefore, if we calculate $\sigma_{j}$ for a given population structure and update rule, we can determine the favoured strategy for any game.

For certain population structures, such as the well-mixed population and the cycle graph, the state is fully described by the number of A-players, $n$. Thus we can define the ratio of fixation probabilities as

$\frac{\rho_{A}}{\rho_{B}}=\prod_{n=1}^{Z-1} \frac{T_{n}^{+}}{T_{n}^{-}}$

where $T_{n}^{ \pm}$are the transition probabilities to go from $n \rightarrow n \pm 1 A$ type individuals (Traulsen et al., 2009). This does not hold in general, as the transition probabilities in more complex population structures will depend on the spatial configurations of different cell types, and thus, are not uniquely defined by $n$. However, it is still possible to utilise this equation, as we see in Sections 2.1.6 and 2.2 , by averaging over possible states to approximate $T_{n}^{ \pm}$.

In the following we consider various cases where the structure coefficients can be calculated from transition probabilities in the weak selection limit, i.e. when $\delta \ll 1$. This limit is commonly employed within evolutionary graph theory in order to obtain analytical results, e.g.(Nathanson et al., 2009; Tarnita et al., 2009a; Allen et al., 2017). Essentially, weak selection implies that the payoffs obtained by playing the game are only a small contribution to overall fitness. It allows expansion of fixation probabilities in powers of the selection strength parameter.

We outline known results for the well-mixed population, the cycle graph with death-birth update rule and $k$-regular graphs with death-birth update rule. We also introduce some new results, deriving the structure coefficients for the cycle graph with birthdeath and shift update rules. Finally, we derive a new approximate expression for the structure coefficients of any population structure with global updating.

\subsubsection{Well-mixed population}

The structure coefficients for a well-mixed population are given by Gokhale and Traulsen (2010):

$\sigma_{j}= \begin{cases}1, & \text { if } 0 \leqslant j \leqslant N-2 \\ \frac{Z-N}{Z}, & \text { if } j=N-1\end{cases}$

(see also Section 2.1.6). Thus we can obtain the condition for $\rho_{A}>\rho_{B}$ by plugging these into Eq. 6 . For a PGG defined by Eq. 1 the condition that cooperators are favoured is

$\frac{Z-N}{Z} b[\beta(1)-\beta(0)]>\sum_{j=0}^{N-1} \sigma_{j} c$.

This becomes

$\frac{b}{c}>\frac{N(Z-1)}{Z-N}$, when we set $\beta(1)=1$ and $\beta(0)=0$. Clearly the shape of the benefit function does not impact whether cooperation is favoured. For a large population this condition becomes $b / c>N$.

\subsubsection{Cycle graph: death-birth update}

We can obtain exact expressions for the structure coefficients of the cycle graph, in the weak selection limit. The cycle is a onedimensional lattice with periodic boundary conditions. Individuals interact with their two nearest-neighbours, thus we have group size $N=3$.

The structure coefficients for the death-birth update rule are derived in Peña et al. (2016). They are given by

$\sigma_{0}=1, \quad \sigma_{1}=Z-2, \quad \sigma_{2}=Z-3$.

From Eq. 6 we obtain the condition for cooperation to be favoured under an NPD, defined by Eq. 3:

$\frac{b}{c}>\frac{3(Z-2)}{2(Z-3)}$

which for $Z \rightarrow \infty$ becomes $b / c>3 / 2$. These conditions are lower than those obtained for a well-mixed population. For a general PGG defined by Eq. 1 we can write down the condition

$\frac{b}{c}>\frac{2(Z-2)}{(Z-3)[\beta(1)+\beta(2 / 3)-\beta(1 / 3)-\beta(0)]}$.

\subsubsection{Cycle graph: birth-death update}

We derive novel results for the birth-death and shift update rules on the cycle, using a similar method to Peña et al. (2016) for the death-birth update rule. For the cycle, the transition probabilities are uniquely defined by the number of $A$-players in the population, $n$. Thus we can write down the ratio of transition probabilities for each $n$. For a birth-death update rule these are

$\frac{T_{n}^{+}}{T_{n}^{-}}= \begin{cases}\left(1+\delta a_{0}\right) /\left(1+\delta b_{1}\right), & \text { if } n=1 \\ \left(1+\delta a_{1}\right) /\left(1+\delta b_{1}\right), & \text { if } 1<n<Z-1 \\ \left(1+\delta a_{1}\right) /\left(1+\delta b_{2}\right), & \text { if } n=Z-1 .\end{cases}$

Substituting these into Eq. 7, and taking the limit, $\delta \ll 1$, we obtain

$\frac{\rho_{A}}{\rho_{B}} \approx 1+\delta\left[a_{0}-b_{2}+(Z-2)\left(a_{1}-b_{1}\right)\right]$.

In order that $\rho_{A}>\rho_{B}$, the second term must be positive. Thus, comparing this condition with Eq. 6, we find the structure coefficients

$\sigma_{0}=1, \quad \sigma_{1}=Z-2, \quad \sigma_{2}=0$.

For the NPD, cooperation is favoured when

$\frac{b}{c}>\frac{3(Z-1)}{Z-3}$

which becomes $b / c>3$ in the large population limit, $Z \rightarrow \infty$. These conditions are equivalent to those obtained for the well-mixed population. For a general PGG defined by Eq. 1 the condition is

$\frac{b}{c}>\frac{Z-1}{(Z-3)[\beta(2 / 3)-\beta(1 / 3)]}$.

\subsubsection{Cycle graph: shift update}

We follow the same procedure to derive the structure coefficients for the shift update rule. This time the ratio of transition probabilities is given by 
$\frac{T_{n}^{+}}{T_{n}^{-}}= \begin{cases}\frac{(Z-1)\left(1+\delta a_{0}\right)}{2\left(1+\delta b_{1}\right)+(Z-3)\left(1+\delta b_{0}\right)}, & \text { if } n=1 \\ \frac{(Z-n)\left(2\left(1+\delta a_{1}\right)+(n-2)\left(1+\delta a_{2}\right)\right)}{n\left(2\left(1+\delta b_{1}\right)+(Z-n-2)\left(1+\delta b_{0}\right)\right)}, & \text { if } 1<n<Z-1 \\ \frac{2\left(1+\delta a_{1}\right)+(Z-3)\left(1+\delta a_{2}\right)}{(Z-1)\left(1+\delta b_{2}\right)}, & \text { if } n=Z-1 .\end{cases}$

In the weak selection limit, $\delta \ll 1$, Eq. 7 becomes

$$
\begin{aligned}
\frac{\rho_{A}}{\rho_{B}} \approx & 1 \\
& \left.+\delta\left[\left(a_{0}-b_{2}\right)+2\left(H_{Z-1}-1\right)\left(a_{1}-b_{1}\right)\right)+\left(Z-2 H_{Z-1}\right)\left(a_{2}-b_{0}\right)\right],
\end{aligned}
$$

where $H_{m}$ is the $m$-th harmonic number:

$H_{m}=\sum_{n=1}^{m} \frac{1}{n}$.

Thus the structure coefficients are given by

$\sigma_{0}=1, \quad \sigma_{1}=2\left(H_{Z-1}-1\right), \quad \sigma_{2}=\left(Z-2 H_{Z-1}\right)$.

The condition for cooperation to be favoured for the NPD is

$\frac{b}{c}>\frac{3(Z-1)}{3(Z-1)-4 H_{Z-1}}$.

In the large population limit this becomes $b / c>1$. As this condition is required in the definition of the NPD, we can state that cooperation is always favoured in the large population limit for a shift update under weak selection.

In fact, if we consider a general cooperation game as defined by Eq. 1, we obtain the condition

$\frac{b}{c}>\frac{1}{\beta(1)-\beta(0)}$

in the large population limit, $Z \rightarrow \infty$. Letting $\beta(1)=1$ and $\beta(0)=0$, we regain the condition $b / c>1$. Thus, for the shift update on the cycle, as with the well-mixed population, the condition for cooperation to be favoured is not dependent on the shape of the benefit function (although in this case we required the large population limit). Furthermore, cooperation is favoured on the cycle with shift update for all PGGs, as defined by Eq. 1, given that the population is sufficiently large.

\subsubsection{Approximate results for $k$-regular graphs}

In higher dimensions the transition probabilities are no longer uniquely defined by the number of $A$-players in the population, but depend also on their configuration. Peña et al. (2016) have derived expressions for the structure coefficients of regular graphs of degree $k \geqslant 3$, with death-birth updating, using pair approximation and diffusion approximation (Ohtsuki et al., 2006b). They compared theoretical predictions with simulation results for the case of a volunteer's dilemma game. They find a good fit for random regular graphs, but that the approximations underestimate the critical benefit-to-cost ratio for lattices.

We do not state the full expressions here which are non-trivial functions of $k$. The condition for cooperation to be favoured with the NPD in the large population limit $(Z \gg k)$ is given by Peña et al. (2016)

$\frac{b}{c}>\frac{k+1}{2}$

\subsubsection{Structure coefficients under global updating}

In the following we derive novel results for the structure coefficients under global updating. We find a general expression which is exact under certain conditions, and provides an approximation for the structure conditions for any population structure with global update rule. The preceding sections have considered games played on a fixed graph or well-mixed population, within groups of fixed size, $N$. For well-mixed populations we were free to choose $N$ (although some results required $N \ll Z$ ), while for regular graphs we set $N=k+1$, where $k$ is the degree of the graph. Here, we relax this condition and allow for variable group size.

We make the assumption that there is a fixed distribution, $f_{j}^{A / B}(n, k)$, defining the probability that an $A / B$-player interacts with $j$ co-players of type $A$, given it has $k$ co-players in total and there are $n$ players of type $A$ in the population. If the population were defined on a graph, this would be the probability of an $A / B-$ player having $j$ A-type neighbours, given $k$ total neighbours. This assumption is true for a well-mixed population or cycle graph, but not necessarily for other population structures where $f_{j}^{A / B}(n, k)$ depends on the specific configuration of players. The frequency of individuals with $k$ neighbours is given by $g_{k}$. We make the further assumptions that this distribution is fixed, and does not depend on type. See Appendix B for a discussion of the validity of this assumption for the VT model.

In general, for a global update rule, we can define the transition probabilities

$T_{n}^{+}=\frac{Z-n}{Z} \frac{n F_{A}}{n F_{A}+(Z-n) F_{B}} \quad T_{n}^{-}=\frac{n}{Z} \frac{(Z-n) F_{B}}{n F_{A}+(Z-n) F_{B}}$,

where

$F_{A}=1+\delta \sum_{k=1}^{Z-1} \sum_{j=0}^{k} f_{j}^{A}(n, k) g_{k} a_{j, k}$

$F_{B}=1+\delta \sum_{k=1}^{Z-1} \sum_{j=0}^{k} f_{j}^{B}(n, k) g_{k} b_{j, k}$

are the population averaged fitnesses. The payoffs $a_{j, k}$ and $b_{j, k}$ depend explicitly on the number of neighbours $k$.

Substituting Eqs. 26-28 into Eq. 7, and taking the weak selection limit we obtain

$\frac{\rho_{A}}{\rho_{B}} \approx 1+\delta \underbrace{\sum_{n=1}^{Z-1} \sum_{k=1}^{Z-1} \sum_{j=0}^{k} g_{k}\left[f_{j}^{A}(n, k) a_{j, k}-f_{j}^{B}(n, k) b_{j, k}\right]}_{\Gamma}$.

Thus $\rho_{A}>\rho_{B}$ when $\Gamma>0$. In the weak selection limit,

$f_{j}^{A}(n, k)=f_{k-j}^{B}(Z-n, k)$

must hold by symmetry, and thus

$\sum_{n=1}^{Z-1} f_{j}^{A}(n, k)=\sum_{n=1}^{Z-1} f_{k-j}^{B}(n, k)$.

Therefore we have

$\Gamma=\sum_{k=1}^{Z-1} \sum_{j=0}^{k} \sum_{n=1}^{Z-1} g_{k} f_{j}^{A}(n, k)\left(a_{j, k}-b_{k-j, k}\right)$.

The condition for $A$ to be favoured over $B$ is thus given by

$\sum_{k=1}^{Z-1} \sum_{j=0}^{k} \sigma_{j, k}\left(a_{j, k}-b_{k-j, k}\right)>0$,

where

$\sigma_{j, k}=g_{k} \sum_{n=1}^{Z-1} f_{j}^{A}(n, k)$

are the structure coefficients. For a fixed group size, $N=k+1$, this reduces to Eq. 6, with

$\sigma_{j}=\sum_{n=1}^{Z-1} f_{j}^{A}(n)$ 
where we have dropped the explicit dependence on $k$.

Recall that this derivation is based on the assumption that $g_{k}$ and $f_{j}^{A}(n, k)$ are fixed. While this is not true in most cases, we can obtain an approximation for the structure coefficients by averaging over a large ensemble of population configurations, i.e. letting $f_{j}^{A}(n)=\left\langle f_{j}^{A}(n)\right\rangle_{0}$. Here, $\langle$.$\rangle represents the mean taken over possible$ configurations and the 0 indicates that these are obtained in the neutral selection limit, i.e. $\delta=0$.

The well-mixed population is an example where $f_{j}^{A}(n)$ is fixed. It is defined by a hypergeometric distribution:

$f_{j}^{A}(n)=\left(\begin{array}{c}Z-1 \\ k\end{array}\right)^{-1}\left(\begin{array}{c}n-1 \\ j\end{array}\right)\left(\begin{array}{c}Z-n \\ k-j\end{array}\right)$.

We can therefore find the structure coefficients (Gokhale and Traulsen, 2010) by substituting this expression for $f_{j}^{A}(n)$ into Eq. 35:

$$
\sigma_{j}=\left(\begin{array}{c}
Z-1 \\
k
\end{array}\right)^{-1} \underbrace{\sum_{n=1}^{Z-1}\left(\begin{array}{c}
n-1 \\
j
\end{array}\right)\left(\begin{array}{c}
Z-n \\
k-j
\end{array}\right)}_{S} .
$$

It can be shown (see Appendix A in Gokhale and Traulsen (2010)) that

$S= \begin{cases}\left(\begin{array}{l}Z \\ k+1\end{array}\right) & \text { if } 0 \leqslant j<k \\ \left(\begin{array}{l}Z-1 \\ k+1\end{array}\right) & \text { if } j=k .\end{cases}$

Thus the structure coefficients are given by

$\sigma_{j}= \begin{cases}\frac{Z}{k+1} & \text { if } 0 \leqslant j<k \\ \frac{Z-k-1}{k+1} & \text { if } j=k .\end{cases}$

These are equivalent to Eq. 8 up to a constant factor. The cycle graph also has a fixed distribution, $f_{j}^{A}(n)$, thus the structure coefficients for the shift update rule can also be obtained exactly using Eq. 35.

For a variable group size the structure coefficients for the wellmixed population are given by

$\sigma_{j, k}=g_{k} \sigma_{j}(k)=g_{k} \begin{cases}\frac{Z}{k+1} & \text { if } 0 \leqslant j<k \\ \frac{Z-k-1}{k+1} & \text { if } j=k,\end{cases}$

where $\sigma_{j}(k)$ are defined in Eq. 39.

As we have seen in previous sections, once the structure coefficients have been determined, we can use Eq. 6 or Eq. 33 to find the condition under which cooperation is favoured. For a PGG defined by Eq. 1 this is given by

$\frac{b}{c}>\frac{Z-1}{\sum_{k=1}^{Z-1} \sum_{j=0}^{k} \sigma_{j, k}\left[\beta\left(\frac{j+1}{k+1}\right)-\beta\left(\frac{k-j}{k+1}\right)\right]}$.

\subsection{Conditions for cooperation to be beneficial under global updating}

Thus far, we have considered conditions under which a mutant is favoured. However, we recall from Section 1.4, that an alternative measure of mutant success can be obtained by considering the conditions under which it is beneficial. Here, we derive the condition for an $A$-mutant to be beneficial, i.e. $\rho_{A}>\rho_{0}$.
As in the previous section, we make the assumption that the distributions $g_{k}$ and $f_{j}^{A / B}(n, k)$ are fixed. Thus the population averaged fitnesses of $A$ and $B$ players are defined by Eqs. 27 and 28 and the transition probabilities by Eq. 26 . The fixation probability for a single $A$-mutant (Traulsen et al., 2009) is then given by

$\rho_{A}=\left[1+\sum_{m=1}^{Z-1} \prod_{n=1}^{m} \frac{T_{n}^{-}}{T_{n}^{+}}\right]^{-1}$.

Substituting in the transition probabilities and taking the weak selection limit $\delta \ll 1$ we obtain

$\rho_{A}=\frac{1}{Z}+\frac{\delta}{Z^{2}} \sum_{k=1}^{Z-1} \sum_{j=0}^{k}\left(\theta_{j, k}^{A} a_{j, k}-\theta_{j, k}^{B} b_{j, k}\right)+\mathcal{O}\left(\delta^{2}\right)$,

where we have defined

$\theta_{j, k}^{A}=g_{k} \sum_{m=1}^{Z-1} \sum_{n=1}^{m} f_{j}^{A}(n, k)$

$\theta_{j, k}^{B}=g_{k} \sum_{m=1}^{Z-1} \sum_{n=1}^{m} f_{j}^{B}(n, k)=g_{k} \sum_{m=1}^{Z-1} \sum_{n=1}^{m} f_{k-j}^{A}(Z-n, k)$.

The final equality is obtained by symmetry arguments in the weak selection limit.

The condition for $A$ to be a beneficial mutation, $\rho_{A}>1 / Z$, is therefore given by

$\sum_{k=1}^{Z-1} \sum_{j=0}^{k}\left(\theta_{j, k}^{A} a_{j, k}-\theta_{j, k}^{B} b_{j, k}\right)>0$.

If we consider a PGG as defined by Eq. 1, then cooperation is beneficial when

$\frac{b}{c}>\frac{Z(Z-1)}{2 \sum_{k=1}^{Z-1} \sum_{j=0}^{k}\left[\theta_{j, k}^{A} \beta\left(\frac{j+1}{k+1}\right)-\theta_{j, k}^{B} \beta\left(\frac{j}{k+1}\right)\right]}$.

For a fixed group size $N=k+1$ these conditions simplify to

$\sum_{j=0}^{k}\left(\theta_{j}^{A} a_{j}-\theta_{j}^{B} b_{j}\right)>0$

and

$\frac{b}{c}>\frac{Z(Z-1)}{2 \sum_{j=0}^{k}\left[\theta_{j}^{A} \beta\left(\frac{j+1}{k+1}\right)-\theta_{j}^{B} \beta\left(\frac{j}{k+1}\right)\right]}$,

where

$\theta_{j}^{A / B}=\sum_{m=1}^{Z-1} \sum_{n=1}^{m} f_{j}^{A / B}(n, k)$.

\section{Public goods games in an epithelium}

A number of studies have considered the evolutionary dynamics of sigmoid PGGs in epithelia, representing the tissue either as a well-mixed population (Archetti, 2013b), or a fixed graph structure with various local update rules (Archetti, 2013c; Archetti, 2016). Here, we use the framework introduced in Renton and Page (2019) to incorporate explicit tissue dynamics, using the Voronoi tessellation (VT) model, with a spatially decoupled (global) update rule. This means that when the population is updated, a 
1 day

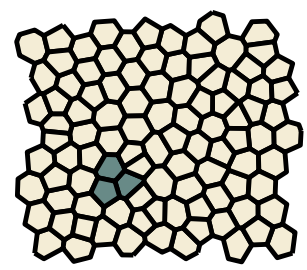

4 days

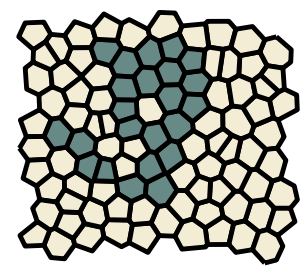

8 days

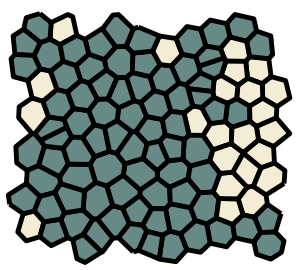

24 days

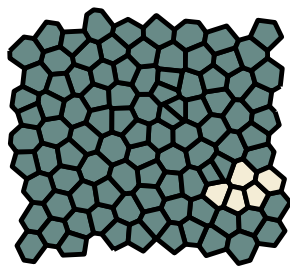

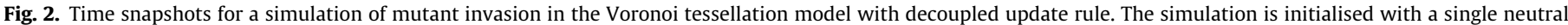

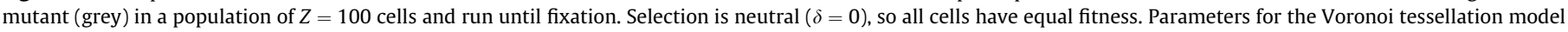
are given in Table 1.

division and death occur simultaneously, but there is no spatial dependence between the two events.

In this section we briefly introduce the VT model, before calculating conditions under which cooperation is favoured and beneficial for a sigmoid PGG. We verify theoretical results by running simulations in various parameter regimes. We also compute the gradient of selection in order to obtain a fuller picture of the dynamics. In all cases we compare VT model results with the well-mixed population.

\subsection{Voronoi tessellation model}

The VT model represents a tissue as a set of points, corresponding to cell centres (Meineke et al., 2001; Van Leeuwen et al., 2009). The shape of each cell, as well as its neighbour connections, is determined by performing a Voronoi tessellation. Cells move subject to spring-like forces, which they exert on their neighbours.

The population evolves through a process of sequential update events, each consisting of a cell division and a cell death, which occur simultaneously. We choose to temporally couple division and death in this way to maintain a constant population size. Allowing separate stochastic birth and death processes, without some other mechanism to maintain homeostatic population size, would result in population extinction or rapid growth. This is something we will address in future work, by introducing contact inhibition as a means of controlling the population size.

Update events occur at rate $\lambda$, according to a continuous time Moran process. When an update occurs, a cell is chosen to divide with probability proportional to fitness. This parent cell is removed from the tissue and replaced with two identical progeny cells, separated by a distance $\epsilon$, across a uniformly random axis. Simultane-

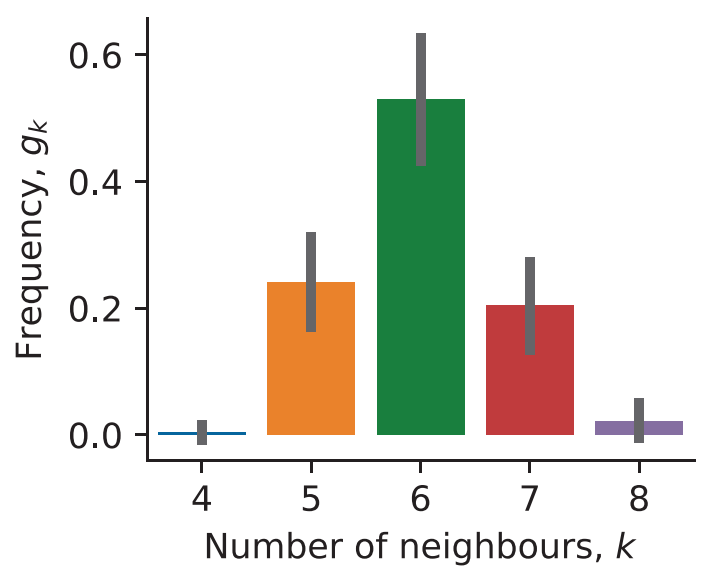

Fig. 3. Degree distribution for the Voronoi tessellation model. Error bars show standard deviation. Data is obtained from simulations with population size, $Z=100$. ously, a cell is chosen to die uniformly at random, and is removed from the tissue. A full description of the VT model used is given in Appendix A.

We obtain $g_{k}$ and $f_{j}^{A}(n, k)$ by averaging over a large ensemble of possible states in the weak selection limit. We then make the assumption that variation around this mean can be neglected. Figs. 3 and 4 , show the distributions $g_{k}$ and $f_{j}^{A}(n, k)$ for the VT model under neutral selection, calculated by averaging over 500 simulations, each of which starts with a single neutral mutant and is run to fixation. An example simulation is shown in Fig. 2. See Appendix B for further discussion on neighbour distributions in the VT model and the validity of assuming $g_{k}$ is independent of $n$ and cell type.

\subsection{Favourable cooperation}

The condition for cooperation to be favoured can be approximated by calculating the structure coefficients using Eq. 34. Fig. 5 plots the VT model structure coefficients with those for a well-mixed population as defined by Eq. 39 .

Using the structure coefficients we can derive the condition for cooperation to be favoured for an arbitrary PGG, as defined by Eq. 1. We define the critical benefit-to-cost ratio $(b / c)_{1}^{*}$, such that $\rho_{C}>\rho_{D}$ when $b / c>(b / c)_{1}^{*}$. Thus, from Eq. 41 we can write

$\left(\frac{b}{c}\right)_{1}^{*}=\frac{Z-1}{\sum_{k=1}^{Z-1} \sum_{j=0}^{k} \sigma_{j, k}\left[\beta\left(\frac{j+1}{k+1}\right)-\beta\left(\frac{k-j}{k+1}\right)\right]}$.

For an NPD, defined by Eq. 3, this becomes

$$
\left(\frac{b}{c}\right)_{1}^{*}=\frac{Z-1}{\sum_{k=1}^{Z-1} \sum_{j=0}^{k} \sigma_{j, k} \frac{2 j+1-k}{k+1}}
$$

Substituting in the structure coefficients we obtain $(b / c)_{1}^{*} \approx 2.22$ for the VT model with decoupled update rule and population size $Z=100$. For a well-mixed population with the same group size distribution we obtain $(b / c)_{1}^{*} \approx 7.35$. As we would expect there is a significant increase in the success of cooperative mutants under the VT model. This is due to the high level of assortment in the VT model, which means cooperators are likely to have more cooperator neighbours than defectors.

On average, cells have six neighbours, thus the mean group size is seven. We can therefore compare the critical benefit-to-cost ratio for a well-mixed population with variable group size, given above, to that of a well-mixed population with fixed group size, $N=7$. The latter is given by Eq. 10 to be $(b / c)_{1}^{*}=7.45$. Clearly, incorporating variation in group size into the well-mixed population has a negligible impact on whether cooperation is favoured. We note however, that the level of variation in group size we have 

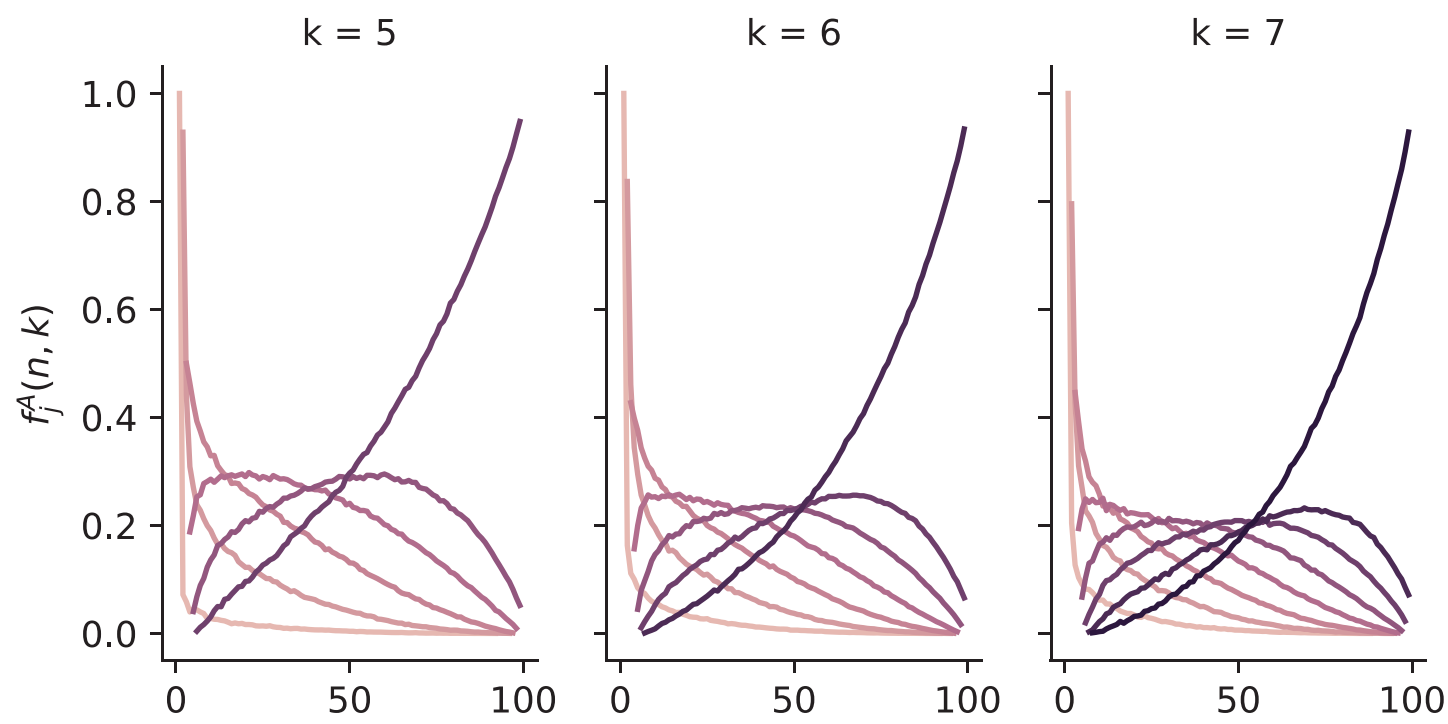

Number of cooperators, $n$
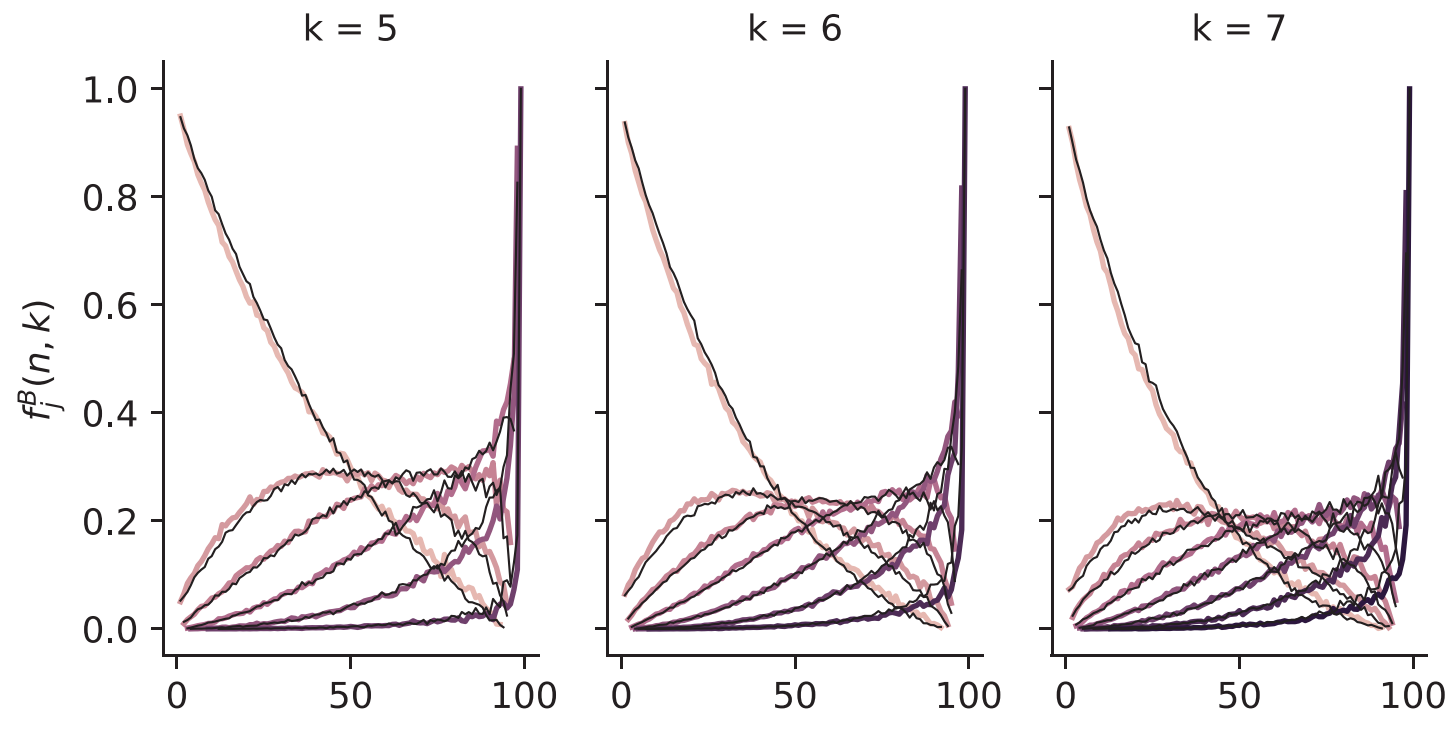

Number of cooperators, $n$

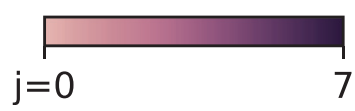

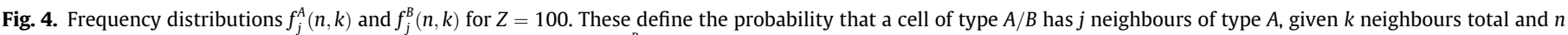

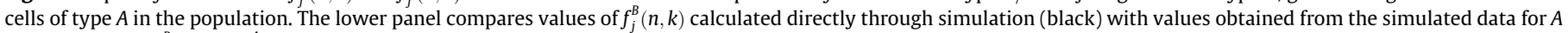
cells defined by $f_{j}^{B}(n, k)=f_{k-j}^{A}(Z-n, k)$.

considered, which is realistic for an epithelium, is small. Larger variation in group size, such as that observed for scale-free networks, may have a larger effect (Archetti, 2016).

We can also use Eq. 51 to determine $(b / c)_{1}^{*}$ for a sigmoid benefit function, defined by Eq. 4. Recall that the logistic function has two parameters: the steepness, $s$, and the inflection point, $h$. Fig. 6 compares the predicted values of $(b / c)_{1}^{*}$ for the VT model, with those for a well-mixed (WM) population with group size 7, and hexagonal lattice (HL) with death-birth update rule. These are obtained from Eq. 51 by using the relevant structure coefficients in each case (structure coefficients for death-birth update on regular graphs are derived in Peña et al. (2016)).
Values of $(b / c)_{1}^{*}$ are symmetric across $h=0.5$ for all three cases, and minimised at $h=0.5$ for the hexagonal lattice and VT model. In Appendix $C$ we show that $(b / c)_{1}^{*}$ is in fact minimised at $h=0.5$, so long as the structure coefficients increase with $j$ for $0 \leqslant j<k$. For the well-mixed population $(b / c)_{1}^{*}$ does not vary with either $s$ or $h$. Furthermore, it is clear for all population types, that as the NPD is approached $(s \rightarrow 0),(b / c)_{1}^{*}$ becomes independent of $h$.

In all parameter regimes, $(b / c)_{1}^{*}$ is highest for the well-mixed population. Both the VT model with decoupled update and HL with death-birth update show similar variation with $s$ and $h$; however, $(b / c)_{1}^{*}$ is always lower for the VT model. Therefore, in terms of 

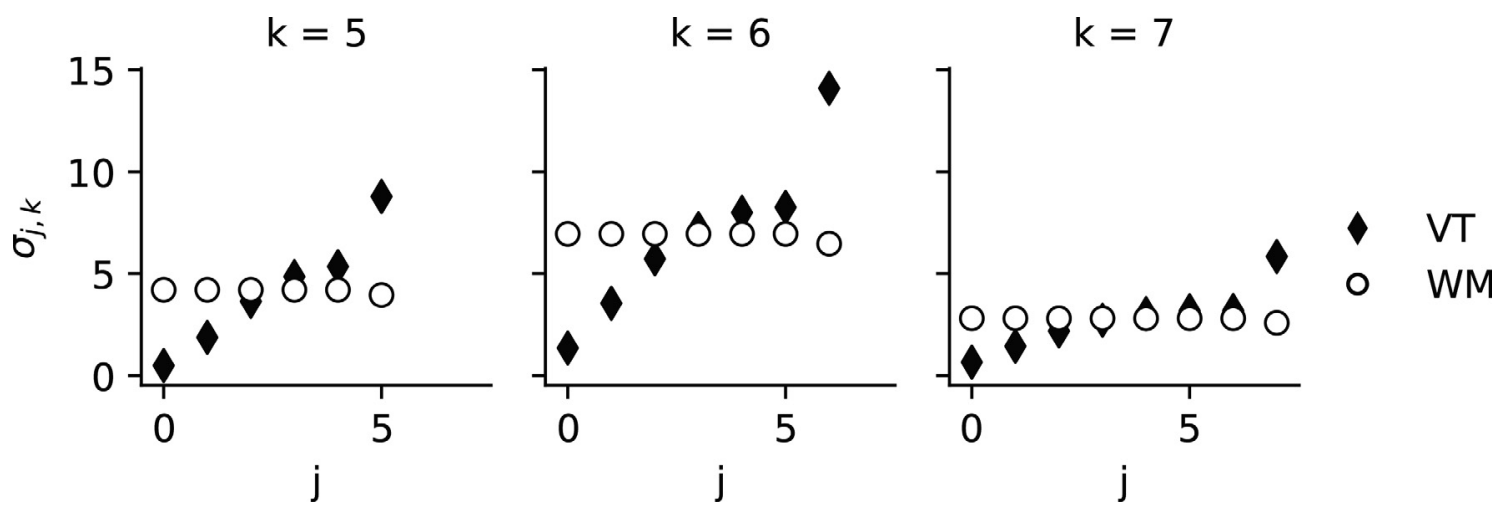

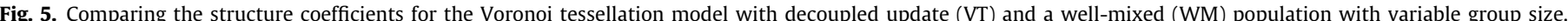

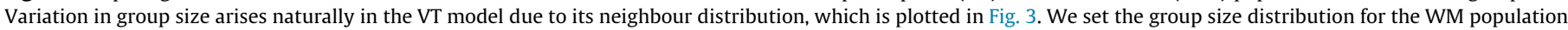
to be equal to that of the VT model. Members of each group are then selected uniformly at random for the WM population.

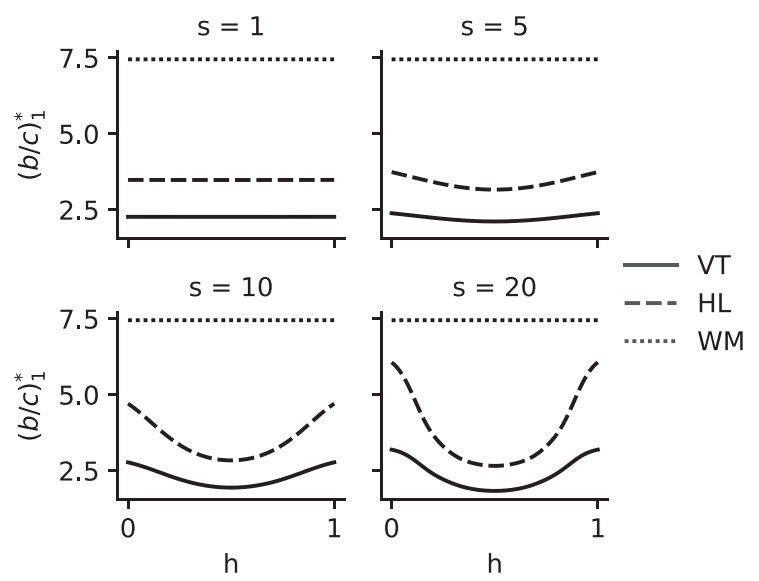

Fig. 6. Comparing the critical benefit-to-cost ratio, $(b / c)_{1}^{*}$ at which $\rho_{C}>\rho_{D}$, for a logistic benefit function. For a well-mixed population with $N=7(\mathrm{WM}),(b / c)_{1}^{*}$ is highest, and independent of the inflection point, $h$, and steepness, $s$. For the Voronoi tessellation model with decoupled update (VT) and fixed hexagonal lattice with death-birth update (HL), $(b / c)_{1}^{*}$ varies with $h$ and $s$. For small $s$ the benefit function approaches linearity and we regain the results for an NPD.

thresholds for favourability, we can determine that cooperation is most successful in the VT model with decoupled update, followed by the hexagonal lattice with death-birth update. Cooperation does least well in the well-mixed population. This suggests that both population structure and global updating promote cooperation.

Fig. 7 (right panel) shows the variation of $(b / c)_{1}^{*}$ with $h$ and $s$ for the VT model. As we have discussed, these results are based on the approximation that $f_{j}^{A}(n, k)$ and $g_{k}$ are fixed. In order to verify the accuracy of this approximation we compare Eq. 51 with simulation results in Fig. 8. Simulated values of $(b / c)_{1}^{*}$ were obtained for each parameter set $(s, h)$ as follows. We calculated $\rho_{C / D}$ for various $b / c$

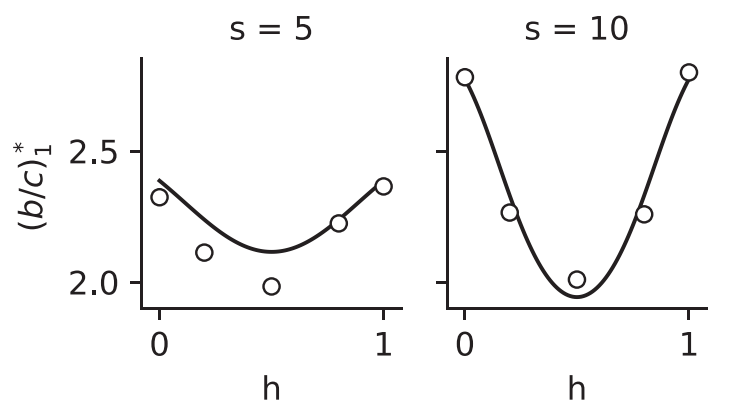

Fig. 8. Critical benefit-to-cost ratio, $(b / c)_{1}^{*}$, above which $\rho_{C}>\rho_{D}$, for a logistic benefit function. The solid line plots Eq. 51 and circles are simulation data. For both $s=5$ and $s=10$ there is symmetry across $h=0.5$, at which point $(b / c)_{1}^{*}$ is minimised.

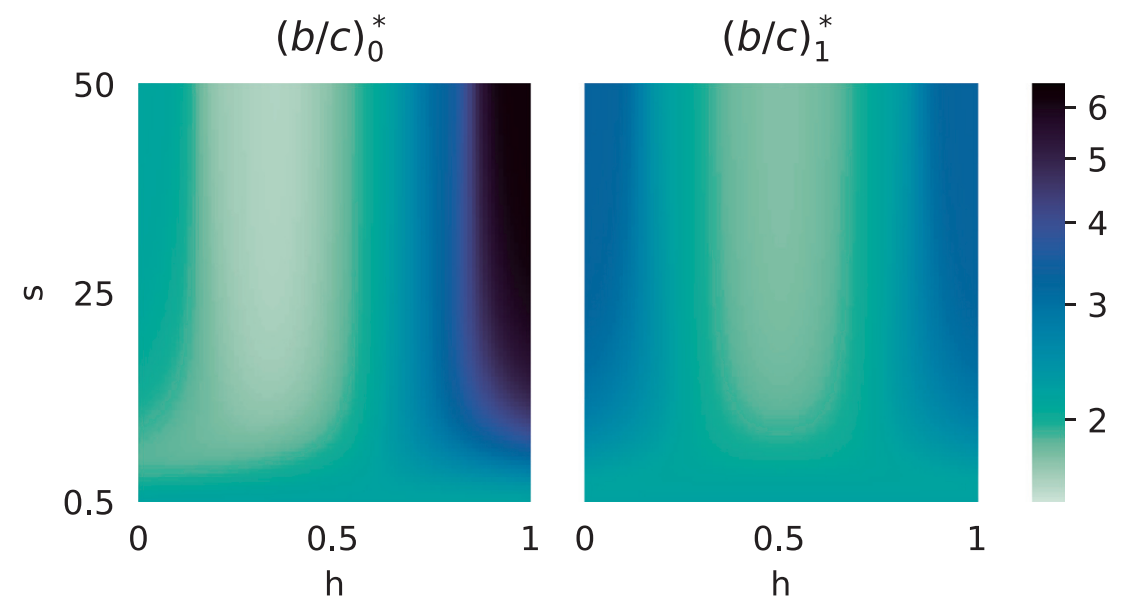

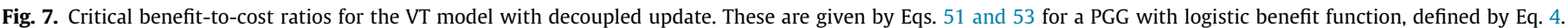

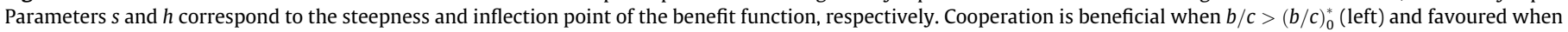
$b / c>(b / c)_{1}^{*}$ (right). 


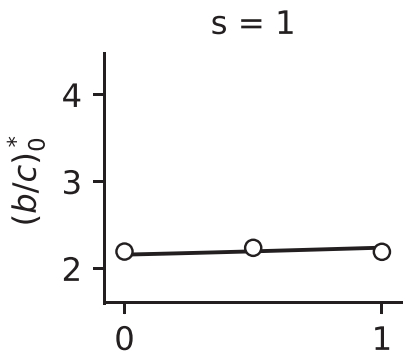

h

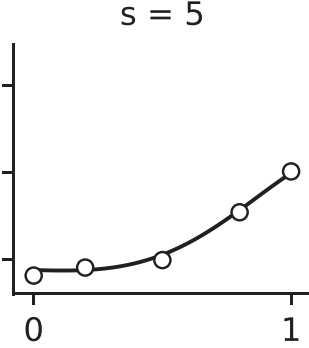

$\mathrm{h}$

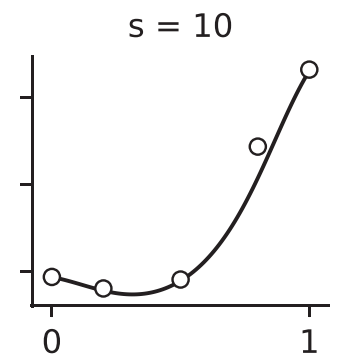

$\mathrm{h}$

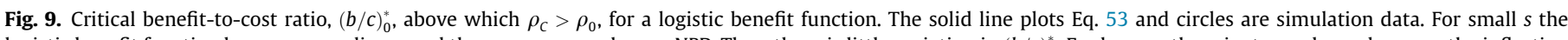

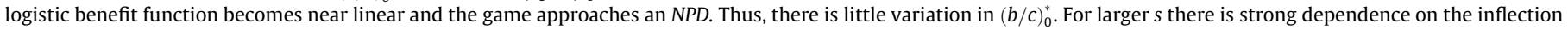
point, $h$, particularly for $h>0.5$.

values, by running $10^{4}$ simulations of the VT model to fixation, starting with a single $C / D$ mutant and population size $Z=100$. In all simulations we use small selection strength $(\delta=0.025)$ and set $c=1$. Thus $(b / c)_{1}^{*}$ is determined by the point at which $\rho_{C}=\rho_{D}$.

There is a decent fit between simulation and theory. It is possible this could be improved by running larger numbers of simulations, however the model is computationally expensive. In any case, the qualitative behaviour is consistent. For a fixed steepness, $s,(b / c)_{1}^{*}$ is minimised at $h=0.5$ and (near) symmetric across this value. The values of $(b / c)_{1}^{*}$ are highest when $h=0$ and $h=1$, where the benefit function provides diminishing returns or increasing returns respectively.

\subsection{Beneficial cooperation}

Thus far we have considered conditions for cooperation to be favoured, i.e. where $\rho_{C}>\rho_{D}$. We can also define the critical benefit-to-cost ratio $(b / c)_{0}^{*}$ above which cooperation is beneficial, i.e. $\rho_{\mathrm{C}}>\rho_{0}$. From Eq. 47 this is given by

$$
\left(\frac{b}{c}\right)_{0}^{*}=\frac{Z(Z-1)}{2 \sum_{k=1}^{Z-1} \sum_{j=0}^{k}\left[\theta_{j, k}^{A} \beta\left(\frac{j+1}{k+1}\right)-\theta_{j, k}^{B} \beta\left(\frac{j}{k+1}\right)\right]},
$$

where $\theta_{j, k}^{A / B}$ are calculated from the distributions $f_{j}^{A / B}(n, k)$ and $g_{k}$ according to Eqs. 44 and 45.

Fig. 7 (left panel) plots $(b / c)_{0}^{*}$ against $s$ and $h$. We can see that for large $s,(b / c)_{0}^{*}$ is maximised at $h=1$ and has a minimum at $h \approx 0.35$. For smaller $s$ this minimum moves towards $h=0$. As $s$ decreases further, the logistic function approaches linear and there is negligible variation in $(b / c)_{0}^{*}$ with $h$. In the limit $s \rightarrow 0$ the game becomes an NPD, with $(b / c)_{0}^{*}=(b / c)_{1}^{*} \approx 2$.2. Fig. 9 compares simu- lated values of $(b / c)_{0}^{*}$ with the theoretical prediction, finding good agreement between the two for a range of $s$ and $h$ values.

We saw in Fig. 6 that the critical benefit-to-cost ratios for cooperation to be favoured, $(b / c)_{1}^{*}$, are lower in the VT model compared to the well-mixed population. Fig. 10 plots $(b / c)_{0}^{*}$ for a well-mixed population with $N=7$ and the VT model with decoupled update, showing clearly that the critical benefit-to-cost ratios for cooperation to be beneficial are also lower for the VT model. Thus under both measures, cooperation is promoted by the VT model. In contrast to $(b / c)_{1}^{*}$, which was independent of the shape of the benefit function for the well-mixed population, $(b / c)_{0}^{*}$ is an increasing function of $h$, so long as $s$ is sufficiently large.

In general, conditions for cooperation to be beneficial are not equivalent to conditions for cooperation to be favoured. This is evident from Fig. 11, where we plot $(\boldsymbol{b} / \boldsymbol{c})_{0}^{*}$ and $(\boldsymbol{b} / \boldsymbol{c})_{1}^{*}$ against $h$ for various values of $s$. The parameter space can be divided into regions where cooperation is both favoured and beneficial, favoured but not beneficial, beneficial but not favoured, and neither favoured nor beneficial.

From Fig. 11 we can see that $(b / c)_{0}^{*}=(b / c)_{1}^{*}$ when $h=0.5$. Furthermore, as $s$ decreases, the regions of parameter space where cooperation is beneficial, but not favoured, or favoured, but not beneficial, get smaller. For sufficiently small $s$ we obtain $(b / c)_{0}^{*} \approx(b / c)_{1}^{*}$. In Appendix D we show that the sigmoid public goods game satisfies a property called antisymmetry-of-invasion when $s \rightarrow 0$ or $h=0.5$. This guarantees that the conditions for a mutant to be beneficial and favoured are equivalent. For both the VT model and well-mixed populations it is clear that behaviour where cooperation is beneficial but not favoured, is only possible when $h<0.5$. Conversely behaviour where cooperation is favoured but not beneficial occurs only when $h>0.5$.

We can understand this intuitively by considering the extreme cases $(h=0,1)$ of the VD game, obtained by letting $s \rightarrow \infty$. When $h=0$, a cooperator always receives the full benefit, even if it has
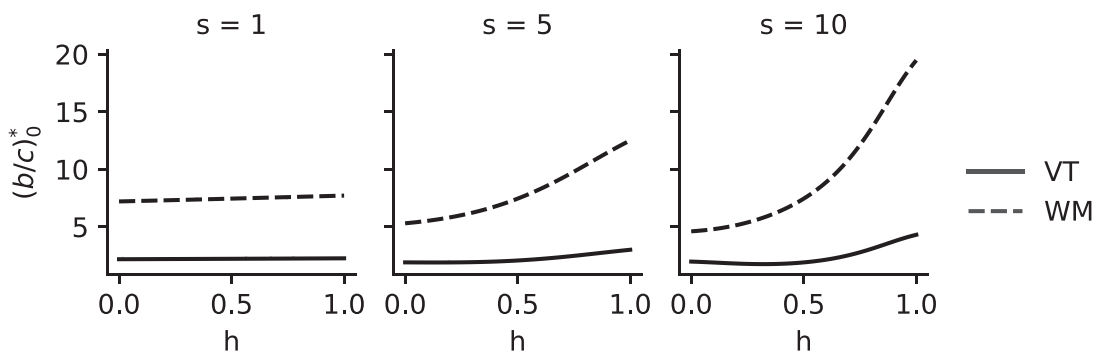

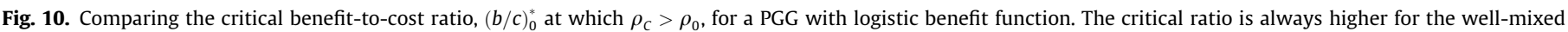

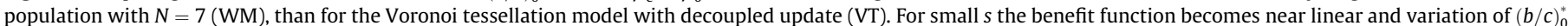

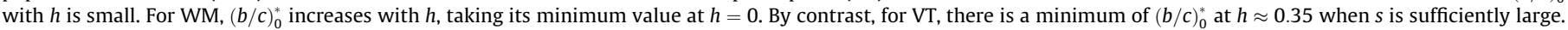
For both WM and VT, $(b / c)_{0}^{*}$ is maximised at $h=1$, for any given $s>0$. 
VT

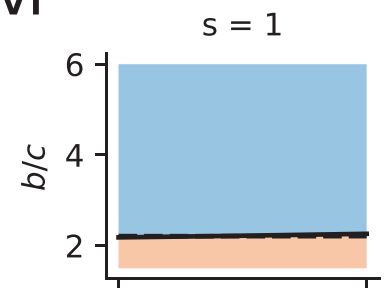

$s=10$

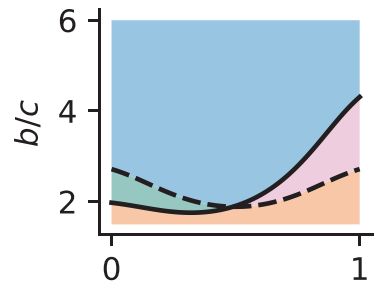

h $s=5$

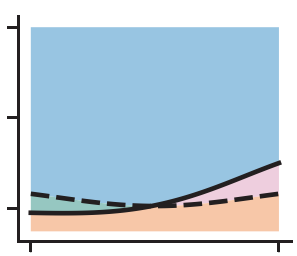

$s=20$

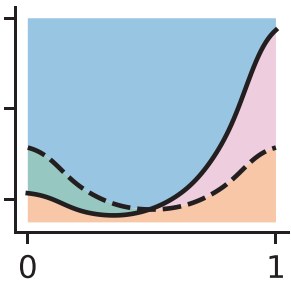

$\mathrm{h}$
WM

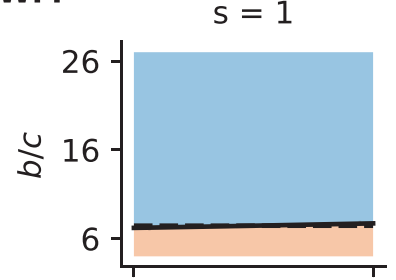

$\mathrm{s}=10$

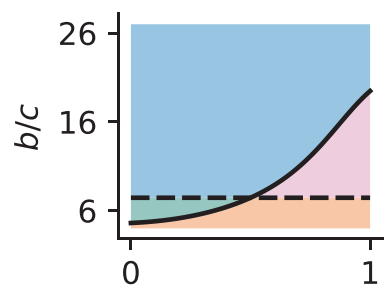

$\mathrm{h}$

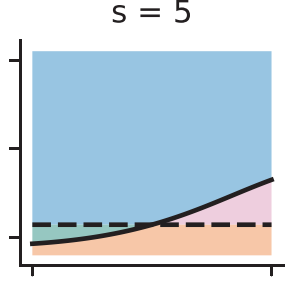

$s=20$

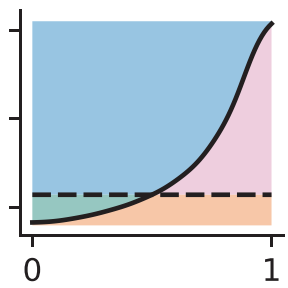

$\mathrm{h}$

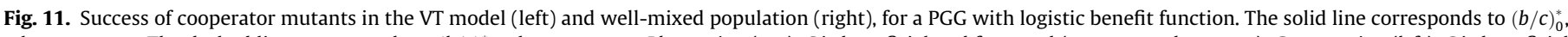

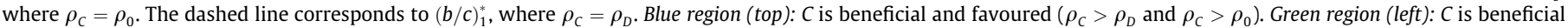

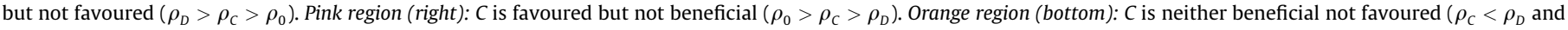
$\left.\rho_{C}<\rho_{0}\right)$.

no cooperator neighbours. Defectors require a single cooperator neighbour to obtain the benefit. Thus, both cooperators and defectors have higher than average fitness early on in the invasion process, when they are most vulnerable to extinction. It is therefore possible, depending on the benefit-to-cost ratio, that both perform better than a neutral invader, and therefore both are beneficial mutations. However, one can still be favoured over the other if its fixation probability is higher.

The converse is true when $h=1$ : defectors will never receive any benefit, and cooperators only obtain the benefit when surrounded by other cooperators. Thus when the number of cooperators/defectors is small, they have lower than average fitness, and there is a high chance they die out early in the invasion process. Therefore, it is possible that neither performs better than a neutral invader.

\subsection{Gradient of selection}

We can obtain more insight into what is happening in the different parameter regions by looking at the gradient of selection, $G(n)=T^{+}(n)-T^{-}(n)$. The transition probabilities are defined by Eq. 26, thus in the weak selection limit, $\delta \ll 1$, the gradient of selection becomes

$G(n) \approx \frac{Z-n}{Z} \frac{n}{Z} \delta\left\{\sum_{k=1}^{Z-1} \sum_{j=0}^{k} g_{k}\left(f_{j}^{A}(n, k) a_{j, k}-f_{j}^{B}(n, k) b_{j, k}\right)\right\}$.

The sum essentially gives the difference in expected payoffs of $A$ and $B$ players. Thus, the right-hand side is identical to that of the replicator equation, which describes the deterministic dynamics obtained in the large-population limit. The sign of $G(n)$ indicates the direction of selection, and we can consider the roots of $G(n)$ as 'fixed points'. Of course, for a finite population there are only two absorbing states, $n=0$ and $n=Z$, however the location of fixed points is still important. In particular, the system may remain for a long time near a stable fixed point. We can classify the behaviour of the system in different parameter regions based on the fixed points of the gradient of selection.

Fig. 12 plots $G(n)$ for a PGG with various values of $h, s$ and $b / c$, both for the VT model and well-mixed population. There are four dynamical regimes, consistent with the deterministic results for PGGs in a well-mixed population in Archetti (2013b):

(i) Dominance: there are only two fixed points at $n=0$ and $n=Z$. Defection dominates if the $n=0$ fixed point is stable, while cooperation dominates if the $n=Z$ fixed point is stable.

(ii) Coexistence: there is an internal stable fixed point, $n_{R}$, along with two unstable fixed points at $n=0$ and $n=Z$. Selection pushes the system towards the stable fixed point, thus it can take a long time to reach one of the absorbing states.

(iii) Coordination: there is an internal unstable fixed point, $n_{L}$, along with two stable fixed points at $n=0$ and $n=Z$.

(iv) Coexistence \& coordination: In addition to the fixed points at $n=0$ (stable) and $n=Z$ (unstable), there is both an unstable internal fixed point on the left, $n_{L}$, and a stable internal fixed point on the right, $n_{R}$. Thus it resembles coexistence, in that there is a stable mixed state; and coordination in that there are two stable fixed points.

These regimes are all familiar in the evolutionary game theory literature for well-mixed populations. The first three correspond to the behaviour of two-player matrix games in well-mixed populations (Traulsen et al., 2009): (i) prisoner's dilemma/harmony game, (ii) snowdrift game, and (iii) stag-hunt game. The final type, coexistence \& coordination, arises for both the N-player stag-hunt (Pacheco et al., 2009) and N-player snowdrift games (Souza et al., 2009).

For the well-mixed population we see dominance when $s$ is sufficiently small, and thus the PGG is approximating an NPD. As expected, cooperation is dominant when $b / c$ is sufficiently high. For higher values of $s$ there is a wide range of behaviour. In a region around $h=0.5$, if $b / c$ is large enough, there are coexistence \& coordination dynamics. There is a large basin of attraction for $n_{R}$ and if the system reaches this fixed point it will spend a long time in the vicinity. However, a single mutant invader must cross $n_{L}$ to reach this, against the selection pressure. As $b / c$ is increased, $n_{L}$ and $n_{R}$ move further apart, increasing the size of the basin of attraction for $n_{R}$. For $h=0.5$, the gradient of selection is symmetric $\left(n_{L}=Z-n_{R}\right)$. 

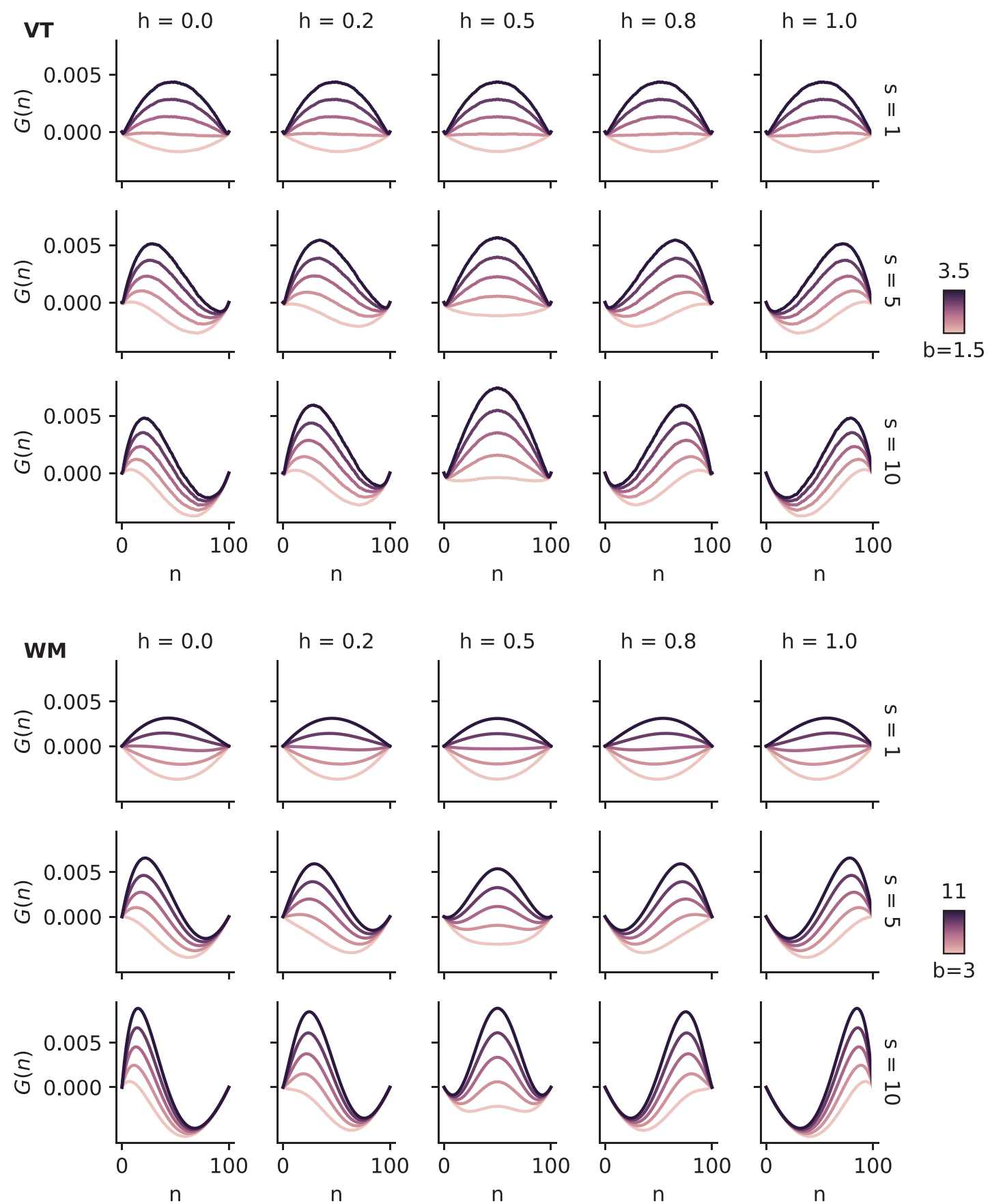

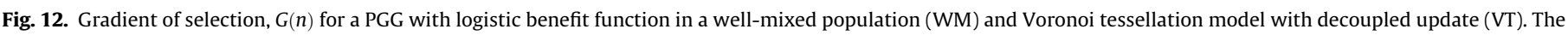

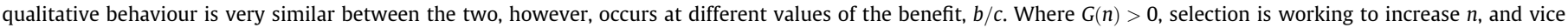
versa. The roots of $G(n)=0$ can be considered as fixed points, and we can use these to classify the behaviour in different parameter regions.

Decreasing $h$ from 0.5 , causes $n_{L}$ and $n_{R}$ to move to the left, eventually entering the coexistence regime. The basin of attraction for the internal stable fixed point is now $0<n<Z$. The system may spend a large amount of time near this point, although it will ultimately end up in one of the absorbing states. In the coexistence regime, as we discussed in Section 3.3 for the VD game with $h=0$, cooperators and defectors have a selective advantage when they are in sufficiently small numbers. This can lead to the case where both are beneficial mutants, and thus cooperation is able to be beneficial but not favoured.

Conversely as $h$ is increased from $0.5, n_{L}$ and $n_{R}$ move to the right and we enter the coordination regime. This corresponds to the region in Fig. 11 where very high values of the benefit-tocost ratio are required for cooperation to be beneficial, even when cooperation is favoured. In Section 3.3 we argued, for the VD game with $h=0.5$, that this is due to the fact that both cooperators and defectors are at a disadvantage when in small numbers. Indeed this is the defining feature of the coordination regime, that $n=0$ and $n=1$ are stable fixed points. Thus, any invader is at a disadvantage initially, as selection pushes it towards dying out. Therefore, it is possible that defectors and cooperators can be at an evolutionary disadvantage compared to a neutral mutant.

The VT model behaviour is qualitatively very similar to that of the well-mixed population. The major difference is that the full 
spectrum of behaviour is available for a much smaller range of $b / c$ values for the VT model. This means that cooperation is successful at smaller benefit-to-cost ratios, as is consistent with our previous findings. It should be noted, however, that these classifications are often approximate for the VT model. We observe, in a number of cases, additional fixed points very close to $n=0$ and $n=Z$. It is also clear from Fig. 12 that the coexistence $\&$ coordination behaviour is much less pronounced that it is for the well-mixed case, with the internal fixed points much closer to the boundaries.

\section{Discussion}

There is an extensive literature on cancer modelling, which goes way beyond evolutionary game theory. For a review, see for example (Altrock et al., 2015). However, evolutionary game theory is increasingly used in cancer modelling (Rockne et al., 2019; Archetti and Pienta, 2019; Wölfl et al., 2020) both to elucidate tumorigenesis (Tomlinson and Bodmer, 1997; Bach et al., 2003; Basanta et al., 2008; Basanta et al., 2008; Archetti, 2016) and to inform potential treatment strategies (Basanta et al., 2012; Kaznatcheev et al., 2017; Zhang et al., 2009; West et al., 2018). Experimental evidence that malignant cells cooperate to drive tumour growth has been found for breast cancer (Marusyk etal., 2014; Cleary et al., 2014) and glioblastoma (Inda et al., 2010. Furthermore, evolutionary games have been explicitly quantified in non-small cell lung cancer Kaznatcheev et al., 2019) and neuroendocrine pancreatic cancer cell cultures (Archetti et al., 2015). These cancers both originate in epithelial cells, of the lung and pancreas, respectively. Disrupting cooperation could therefore be important for improving cancer treatment (Zhou et al., 2017).

Many models of cancer evolution assume populations of cells to be well-mixed (Basanta et al., 2012; Archetti, 2013b; Gerlee and Altrock, 2017; West et al., 2018). However, the importance of spatial structure is increasingly recognised, even for simple mutations (Waclaw et al., 2015; West et al., 2021). Population structure has long been established as a mechanism for promoting the evolution of cooperation (Nowak, 2006). If interactions are limited to an individual's neighbourhood, then cooperators can form mutually beneficial clusters. However, the success of cooperation is also influenced by the update rule. Results for the cycle graph in Section 2.1 demonstrate that a global update rule, such as the shift update, can lead to less stringent conditions for cooperation to be favoured when compared to local update rules. Within the local update rules there are also clear differences: cooperation tends to fare better with a death-birth update rule than a birth-death. In fact for the birth-death update on a cycle, the condition for cooperation to be favoured under an NPD game is equivalent to the well-mixed population. Thus the benefits of clustering are negated in this case.

It is therefore important to take into account realistic population structure and update dynamics of the tissue or tumour when considering the evolution of cooperation amongst cells. Our focus has been to consider how global updating affects the evolution of cooperation in a population structure representative of an epithelium. We have used the VT model to represent an epithelium, which allows death and division to be implemented independently, and therefore, it is trivial to implement what we call the decoupled update rule (Renton and Page, 2019). We chose to focus on global updating, as it presents the opposite extreme to local update rules which have been extensively studied within evolutionary graph theory (Zukewich et al., 2013; Allen et al., 2017; Peña et al., 2016). Furthermore, we have been able to derive quasi-analytical results, which could be applied to other population models that use global updating. Our results are general for multiplayer games; however, we have focused on the analysis of sigmoid public goods games, as it has been proposed that they provide good models for the production of diffusible growth factors.

We have demonstrated that, for a sigmoid PGG, cooperation is more successful in the VT model compared to a well-mixed population. In both cases, the evolutionary outcomes depend on the parameters $s$ and $h$ of the logistic benefit function, as well as the benefit-to-cost ratio. In general, a lower benefit-to-cost ratio is required for cooperative success for the VT model, than the wellmixed population. In other words, cells need a lower incentive to cooperate. This is consistent with our expectations: both models use global updating, however the population structure in the VT model allows for positive assortment of cooperators.

Although cooperation is more successful in the VT model, than the well-mixed population, the qualitative behaviour is very similar. We have characterised the evolutionary dynamics by considering conditions for which cooperation is beneficial and/or favourable, as well as calculating the gradient of selection.

As long as the steepness, $s$, is large enough, we tend to see coexistence behaviour when the inflection point, $h$, is less than a half and coordination behaviour when it is greater. These regimes are characterised by the fixed points of the gradient of selection. They also correspond to the regions in parameter space where cooperation is beneficial, but not favourable (coexistence), and favourable, but not beneficial (coordination). For small steepness, the game approaches an NPD and there is dominance behaviour. In this regime, conditions for cooperation to be beneficial and favoured coincide.

Examining the gradient of selection enables us to identify an additional dynamical regime: mixed coexistence \& coordination, which occurs in a region around $h=0.5$, as long as $s$ and $b / c$ are sufficiently large. This regime is characterised by two stable fixed points, one corresponding to all-defection, and the other to a heterogenous, majority-cooperator state. This dynamic has been identified previously for both well-mixed populations (Archetti, 2013b) and graph-structured populations with local updating (Archetti, 2016). We have shown that it can also occur for the VT model, however the internal fixed points tend to be much closer to the boundaries.

It is beyond the scope of this paper to consider the full dynamics for an epithelial population structure with local update rules. However, we have considered conditions for cooperation to be favourable on a hexagonal lattice with death-birth update rule using results from Peña et al. (2016). We found the critical benefit-tocost ratios to be intermediate between the well-mixed population and VT model. This is consistent with previous results for twoplayer games (Renton and Page, 2019). Taken together, these results suggest not only that population structure promotes cooperation, but that global updating also plays a crucial role. We can thus consider a general rule for cooperation is that it prefers local game play but global competition for offspring.

It is worth taking a moment to consider the implications of beneficial and favourable mutations for invasion, and how we distinguish between the two concepts. Whether or not a mutation is beneficial is perhaps the most relevant measure for a single invasion event. It essentially tells us that the mutated cell has a higher probability of invasion in a wild-type population than a wild-type cell would have, and therefore it has an evolutionary advantage. The significance of a mutation being favourable is a little less clear, as it compares two different invasion processes: the probability of invasion of a mutated cell in a wild-type population is higher than the converse scenario, where a wild-type cell invades a population of mutants. However, the condition for a mutant to be favoured is also equivalent to the condition for cooperation to dominate, if mutation is allowed. 
These results suggest that the population structure, the update rule and the game all play important roles in determining the evolutionary success of cooperation. Cancer models which utilise evolutionary games (You et al., 2017; Gatenby and Brown, 2020) may therefore underestimate the success of cooperative phenotypes, if they fail to account for population structure or assume that death and division are more tightly coupled than is realistic. For example, therapeutic strategies that aim to eliminate cooperation by manipulating evolutionary dynamics, rely on accurate predictions of those dynamics (Archetti, 2013a).

We do not suggest that our regime, with fully local interactions and fully global competition is necessarily realistic for invasion processes in tissues. We have assumed that cells only interact with their immediate neighbours; however, interaction groups may be much larger and likely depend on the specific context. For example, in the case of growth factor production, group size will depend on diffusion range. Estimates of these diffusion ranges are difficult to obtain experimentally (Archetti et al., 2017). However, larger group sizes tend to suppress cooperation (Archetti and Scheuring, 2012), so it is an important consideration.

We chose to focus on global updating, as it presents the opposite extreme to a local update rule. It is likely, however, that update dynamics in a real epithelium lie somewhere in between. Contact inhibition (McClatchey and Yap, 2012), and other densitydependent processes (Eisenhoffer et al., 2012; FernandezGonzalez and Zallen, 2012), result in spatial coupling between death and division, which is likely tissue dependent. Stronger contact inhibition could result in dynamics closer to the death-birth update (Mesa et al., 2018), while weaker contact inhibition is closer to global updating.

Interestingly, loss of contact inhibition is associated with malignancy (McClatchey and Yap, 2012), suggesting that updating is more global, and thus cooperation could be more successful, than in healthy tissues. In future work, we will consider the effect of contact inhibition on cooperation, and the spectrum of behaviour between local and global updating. Understanding the nature of spatial coupling in real epithelia, or in cancerous tumours, could be crucial for predicting evolutionary outcomes.

Our general conclusion that local game play and global competition for offspring favour cooperation has implications beyond applications to cancer, where cooperation unusually may be considered undesirable. In a societal context, where cooperation is desirable, it may be promoted by engineering an environment rich in local social interactions, which nevertheless allows for imitation of successful strategies more globally.

\section{Data accessibility}

The code and data can be accessed at https:// github.com/jessierenton/pgg-epithelium.

\section{CRediT authorship contribution statement}

Jessie Renton: Conceptualization, Methodology, Software, Formal analysis, Writing - original draft. Karen M. Page: Conceptualization, Formal analysis, Writing - review \& editing, Supervision.

\section{Declaration of Competing Interest}

The authors declare that they have no known competing financial interests or personal relationships that could have appeared to influence the work reported in this paper.

\section{Acknowledgments}

This research was funded by an EPSRC studentship held by JR.
Table 1

Table of parameters used in the Voronoi tessellation model (Osborne et al., 2017; Renton and Page, 2019).

\begin{tabular}{lll}
\hline Parameter & Description & Value \\
\hline$\mu$ & spring constant & 50 \\
$s$ & natural cell separation & 1 \\
$\epsilon$ & initial sibling cell separation & 0.1 \\
$\eta$ & drag coefficient & 1 \\
$\Delta t$ & time-step $(\mathrm{h})$ & 0.005 \\
$\lambda$ & rate of division/death $\left(\mathrm{h}^{-1}\right)$ & $12^{-1}$ \\
\hline
\end{tabular}

\section{Appendix A. Voronoi tessellation model}

The Voronoi tessellation (VT) model was introduced in Meineke et al. (2001) and Van Leeuwen et al. (2009). We use the version and parameter values from Renton and Page (2019) in all simulations in this paper. Parameters are given in Table 1 and the model is defined as follows.

The VT model represents a tissue as a set of points in a fixed domain with periodic boundary conditions. Each point corresponds to a cell-centre and moves subject to spring-like forces that cells exert on their neighbours.

We define the force cell $j$ exerts on its neighbour $i$ to be

$\boldsymbol{F}_{i j}(t)=-\mu \frac{\boldsymbol{r}_{i j}(t)}{\left|\boldsymbol{r}_{i j}(t)\right|}\left(\left|\boldsymbol{r}_{i j}(t)\right|-s_{i j}(t)\right)$,

where $\mu$ is the spring constant, $\boldsymbol{r}_{i j}=\boldsymbol{r}_{i}-\boldsymbol{r}_{j}$ is the vector pointing from cell $j$ to cell $i$, and $s_{i j}$ is the natural separation between cells $i$ and $j$. This is set be a constant $s_{i j}=s$, with the exception that newborn sibling cells have a separation $\epsilon$ immediately after division. For these cells, $s_{i j}$ grows linearly over the course of an hour to reach $s$. The total force on a cell $i$ is given by

$\boldsymbol{F}_{i}(t)=\sum_{j \in \mathscr{N}_{i}(t)} \boldsymbol{F}_{i j}$

where $\mathscr{N}_{i}(t)$ denotes the set of cells neighbouring $i$.

It is assumed that motion is over-damped and thus the equation of motion for $i$ is the first order differential equation

$\eta \frac{d \boldsymbol{r}_{i}}{d t}=\boldsymbol{F}_{i}(t)$

where $\eta$ is the damping constant. This is solved numerically using

$\boldsymbol{r}_{i}(t+\Delta t)=\boldsymbol{r}_{i}(t)+\frac{\Delta t}{\eta} \boldsymbol{F}_{i}$,

where the time-step, $\Delta t$, is sufficiently small to ensure numerical stability.

At each time-step, after cells have moved, a Voronoi tessellation is performed. This partitions the domain into polygonal regions, each corresponding to the shape of a cell. It also defines the neighbourhood connections, which are needed to determine the forces cells exert on one another, as well as cell fitnesses.

Cell division is implemented within the VT model, by removing the parent cell and replacing it with two progeny cells, separated by a distance $\epsilon$, across a uniformly random axis. Cell death simply requires the dead cell to be removed. After a death or division, Voronoi tessellation must be performed to obtain new neighbour connections.

\section{Appendix B. Neighbour distributions in the VT model}

In Sections 2.1 and 2.2 we derived conditions under which cooperation is favoured and beneficial, given by Eqs. 41 and 47, respectively. These derivations are based on the assumption that the frequency of cells with $k$ neighbours is a fixed distribution, 

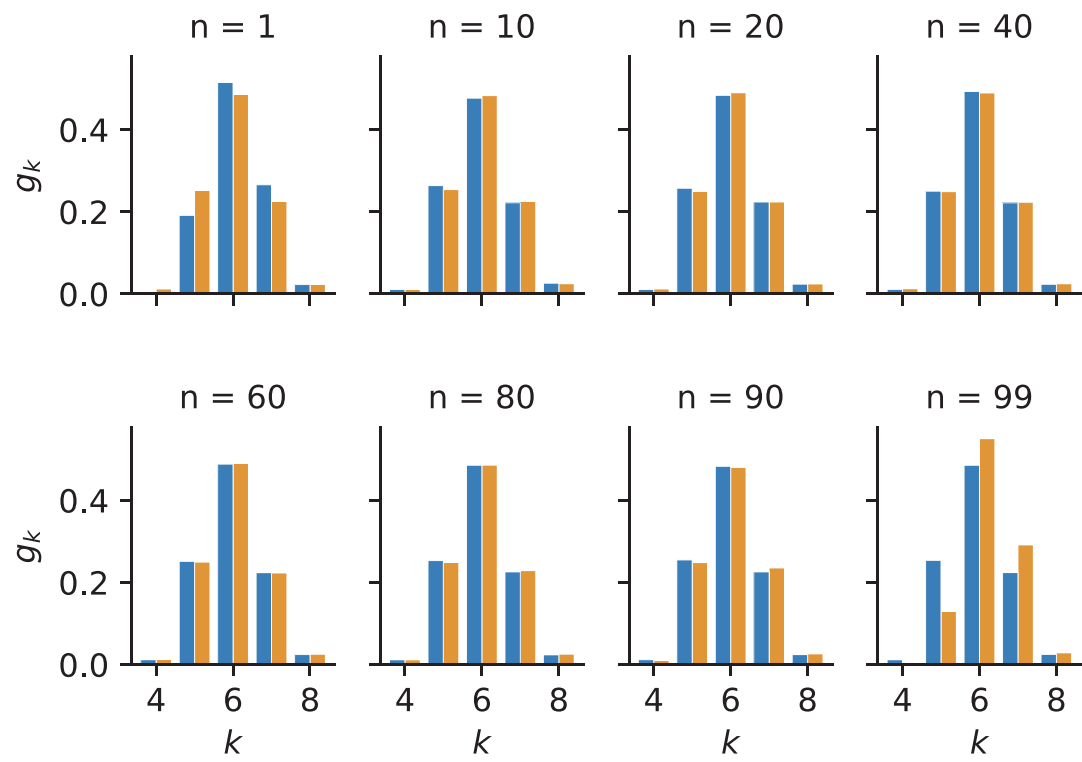

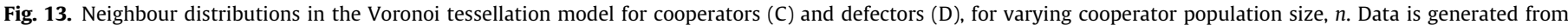
simulations with total population size $Z=100$ in the neutral selection limit, $\delta=0$.

$g_{k}$, independent of the cell type or the number of cooperators in the population, $n$.

Fig. 13 plots neighbour distributions from simulations of the VT model for cooperators and defectors at different values of $n$. It is clear from the plot that the assumption is a reasonable one. The neighbour distributions are approximately equal for different values of $n$ and for the two cell types. The exception is when there are either very few cooperators or very few defectors, i.e. near $n=1$ and $n=99$ respectively. In the case where there is only one or very few cooperators, the cooperator neighbour distribution becomes slightly more narrow. The converse is true when there are few defectors.

\section{Appendix C. Minimising the critical benefit-to-cost ratio at which cooperation is favoured}

In Section 2.2 we considered conditions for cooperative success for a sigmoid benefit function $\beta(x, h)$, as defined by Eq. 4 . Here, we have made explicit the dependence on the inflection point. It is clear from Fig. 6 that the critical benefit-to-cost ratios, $(b / c)_{1}^{*}$, at which $\rho_{C}=\rho_{D}$, are minimised at $h=0.5$, and symmetric across that point. This appears to hold for both the Voronoi tessellation model with decoupled update, and for the death-birth update on a fixed hexagonal lattice. In the following we show that this is indeed true for any system where $0<s<\infty$ and the structure coefficients, $\sigma_{j}$, are increasing for $0 \leqslant j<k$.

We rewrite Eq. 51 , defining $(c / b)_{1}^{*}$, such that cooperation is favoured for $c / b<(c / b)_{1}^{*}$

$\left(\frac{c}{b}\right)_{1}^{*}=\frac{1}{Z-1} \sum_{j=0}^{k} \sigma_{j}\left[\beta\left(\frac{j+1}{k+1} ; h\right)-\beta\left(\frac{k-j}{k+1} ; h\right)\right]$.

We have assumed that the number of neighbours, $k$, is fixed; however, the results are easily generalisable to variable $k$. Defining

$\gamma_{j}(h)=\beta\left(\frac{j+1}{k+1} ; h\right)-\beta\left(\frac{k-j}{k+1} ; h\right)$

we obtain
$\left(\frac{c}{b}\right)_{1}^{*}=\frac{1}{Z-1}\left[\sigma_{k}+\sum_{k>j \geqslant k / 2}\left(\sigma_{j}-\sigma_{k-j-1}\right) \gamma_{j}(h)\right]$.

By taking derivatives with respect to $h$ we show that for $k / 2 \leqslant j<k, \gamma_{j}(h)$ is maximised when $h=0.5$. In order that this corresponds to a unique maximum of $(c / b)_{1}^{*}$, and thus a minimum of the critical benefit-to-cost ratio, certain conditions on $\sigma_{j}$ must be satisfied.

First we show that $\gamma_{j}$ has one extremum at $h=0.5$ for $0<s<\infty$. We substitute Eq. 4 into Eq. 60 and take the first derivative with respect to $h$, letting $r=\frac{j+1}{k+1}$. Thus we obtain

$$
\begin{aligned}
\frac{d \gamma_{j}}{d h} & =\frac{d}{d h}\left[\frac{\left(1+e^{s(h-r)}\right)^{-1}-\left(1+e^{s(h+r-1)}\right)^{-1}}{\left(1+e^{s(h-1)}\right)^{-1}-\left(1+e^{s h}\right)^{-1}}\right] \\
& =\frac{d}{d h}\left[\frac{e^{s(r-1)}-e^{-s r}}{1-e^{-s}} \cdot \frac{\left(1+e^{s(h-1)}\right)\left(1+e^{s h}\right)}{\left(1+e^{s(h-r)}\right)\left(1+e^{s(h+r-1)}\right)}\right] \\
& =s \cdot \frac{e^{s(r-1)}-e^{-s r}}{1-e^{-s}} \cdot \frac{e^{s h}\left(1+e^{-s}-e^{-s r}-e^{s(r-1)}\right)\left(1-e^{s(2 h-1)}\right)}{\left(1+e^{s(h+r-1)}\right)^{2}\left(1+e^{s(h-r)}\right)^{2}} .
\end{aligned}
$$

Setting $d \gamma_{j} / d h=0$, gives one root at $h=0.5$, for $0<s<\infty$. This is a unique stationary point of $(c / b)_{1}^{*}$ so long as there is at least one value of $j \in[k / 2, k)$ for which $\left(\sigma_{j}-\sigma_{k-j-1}\right) \neq 0$. We can show that this is a maximum by considering the second derivative at $h=0.5$

$\left.\frac{d^{2} \gamma_{j}}{d h^{2}}\right|_{h=\frac{1}{2}}=-2 s^{2} \cdot \frac{e^{s / 2}\left(1+e^{-s}-e^{-s r}-e^{s(r-1)}\right)\left(e^{s(r-1)}-e^{-s r}\right)}{\left(1-e^{-s}\right)\left(1+e^{s(r-1 / 2)}\right)^{2}\left(1+e^{-s(r-1 / 2)}\right)^{2}}$

which is negative given that $1 / 2<r<1$. This corresponds to $(k-1) / 2<j<k$, encompassing all the values of $j$ which we sum over in Eq. 61. Therefore, in order that $(c / b)_{1}^{*}$ is maximised when $h=0.5$, we require that $\left(\sigma_{j}-\sigma_{k-j-1}\right) \geqslant 0$ for $k / 2 \leqslant j<k$ and nonzero for at least one value of $j$ in the range. This condition is guaranteed if $\sigma_{j}$ is an increasing, but not constant function for $0 \leqslant j<k$.

It is clear from Fig. 5 that $\sigma_{j+1, k}>\sigma_{j, k} \forall j, k$ for the VT model with decoupled update, and therefore, $h=0.5$ maximises $(c / b)_{1}^{*}$ in this case. For $k$-regular graphs with death-birth update rule, we can 


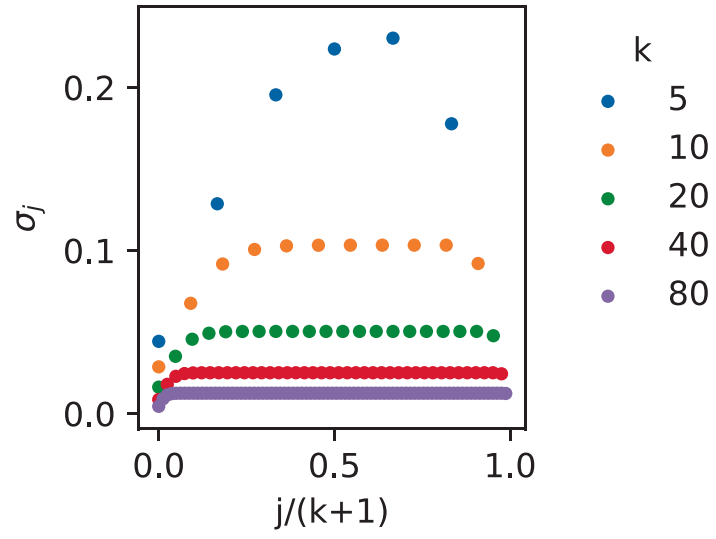

Fig. 14. Structure coefficients, $\sigma_{j}$, for $k$-regular graphs with death-birth update rule (Peña et al., 2016). It is clear that $\sigma_{j}$ is increasing (or constant) for $j<k$.

verify whether this is true by using the approximate expressions for the structure coefficients derived in Peña et al. (2016). These are plotted for various $k$ values in Fig. 14. For smaller values of $k$, we can see that $\sigma_{j}$ is strictly increasing for $0 \leqslant j<k$. However, as $k$ increases, a growing region appears for which $\sigma_{j}$ is constant. So long as there is at least one value of $j<k$ for which $\left(\sigma_{j}-\sigma_{k-j-1}\right)>0,(c / b)_{1}^{*}$ is maximised at $h=0.5$. However, as $k \rightarrow \infty$, we approach the case where $\sigma_{j}$ are constant for $j<k$, and we regain the well-mixed population result that $(c / b)_{1}^{*}$ is independent of $h$.

Thus far we have limited ourselves to the case where $0<s<\infty$. In the limit $s \rightarrow 0$, we obtain an NPD game with a linear benefit function which is independent of $h$. The value of $(c / b)_{1}^{*}$ therefore does not depend on $h$ either, as can be seen in Fig. 6 . In the limit $s \rightarrow \infty$, the VD game is approached and the benefit function ceases to be continuous. In this case the unique maximum at $h=0.5$ is maintained only if $\sigma_{j}$ are strictly increasing, and therefore $\left(\sigma_{j}-\sigma_{k-j-1}\right)>0$. This is true for the VT model with decoupled update and for $k$-regular graphs with death-birth update, if $k$ is sufficiently small. On the other hand, if $\left(\sigma_{j}-\sigma_{k-j-1}\right)=0$ for some values of $j \in[k / 2, k), h=0.5$ ceases to be an isolated maximum, and there is a region of $h$ values, around $h=0.5$, which maximise $(c / b)_{1}^{*}$.

\section{Appendix D. Equivalence of beneficial and favoured mutants}

\section{D.1. Antisymmetry-of-invasion property}

In Sections 2.1.6 and 2.2 we derived the conditions under which a mutant is beneficial or favoured, respectively, for a global update rule. Here, we show that these conditions are equivalent if the payoffs satisfy a property we call antisymmetry-of-invasion. We consider multiplayer games with fixed group size. However, the results can be generalised to variable group size, given certain conditions.

The values $\theta_{j}^{A}$ and $\theta_{j}^{B}$, defined by Eq. 50 , can be written as

$\theta_{j}^{A}=\sum_{n=1}^{Z-1}(Z-n) f_{j}^{A}(n)$

$\theta_{j}^{B}=\sum_{n=1}^{Z-1} n f_{k-j}^{A}(n)$.

Thus we have

$\theta_{j}^{A}+\theta_{k-j}^{B}=Z \sum_{n=1}^{Z-1} f_{j}^{A}(n)=Z \sigma_{j}$, where the last equality is from the definition of $\sigma_{j}$ as stated by Eq. 35. The condition for A to be beneficial, given by Eq. 48, thus becomes

$\sum_{j=0}^{k}\left[\theta_{j}^{A} a_{j}-\left(Z \sigma_{j}-\theta_{j}^{A}\right) b_{k-j}\right]>0$.

This can be rewritten in the form

$\sum_{j=0}^{k}\left(\theta_{j}^{A}-\frac{Z}{2} \sigma_{j}\right)\left(a_{j}+b_{k-j}\right)+\frac{Z}{2} \sum_{j=0}^{k} \sigma_{j}\left(a_{j}-b_{k-j}\right)>0$.

If the payoffs satisfy

$a_{j}+b_{k-j}=Q$,

where $Q$ is a constant that is independent of $j$, then the first term in Eq. 69 vanishes. The condition for $A$ to be beneficial, therefore, becomes

$\sum_{j=0}^{k} \sigma_{j}\left(a_{j}-b_{k-j}\right)>0$

which is equivalent to the condition for $A$ to be favoured, as defined by Eq. 6 . Thus the conditions for cooperation to be beneficial and favoured are equivalent when Eq. 70 holds, which we call the antisymmetry-of-invasion property. If $Q$ is independent of $k$, this result generalises to variable group size.

\section{D.2. Implications of antisymmetry-of-invasion}

In games which satisfy antisymmetry-of-invasion, defined by Eq. 70, there is a fixed total payoff that can be obtained when equal numbers of $A$ and $B$ co-players are distributed between an $A$ and $B$ player. By this we mean that the $A$-player has $j$ other $A$-players in its group and $k-j B$-players, whilst the $B$-player has $j$ other $B$ players, and $k-j A$-players. Regardless of how the co-players are distributed (the value of $j$ ), the sum of the payoffs to the $A$ and $B$ player are the same.

The implications of this property can be better understood if we consider symmetric invasion processes. Consider, for example, an arbitrary evolutionary path through the state space. This path can be represented by a sequence of states

$S=\left(G_{0}, \boldsymbol{s}_{0}\right) \rightarrow\left(G_{1}, \boldsymbol{s}_{1}\right) \rightarrow \ldots \rightarrow\left(G_{L}, \boldsymbol{s}_{L}\right)$,

where $G_{q}$ are graphs representing the population structure at time $t_{q}$ and $\boldsymbol{s}_{q}$ are $Z$-dimensional vectors giving the type of each individual at time $t_{q}$. Thus, $\left[\boldsymbol{s}_{q}\right]_{i}=1$ if the $i$ th individual is an $A$-player and $\left[\boldsymbol{s}_{q}\right]_{i}=0$ if it is a $B$-player. Recall $Z$ is the population size. There are $L$ transitions between states, each of which is caused by an update event (i.e. a death and a division).

The symmetric invasion process $\tilde{S}$ is obtained by flipping the type of each individual $(A \rightarrow B$ and $B \rightarrow A)$, as illustrated in Fig. 15. Thus

$\tilde{S}=\left(G_{0}, \tilde{\boldsymbol{s}}_{0}\right) \rightarrow\left(G_{1}, \tilde{\boldsymbol{s}}_{1}\right) \rightarrow \ldots \rightarrow\left(G_{L}, \tilde{\boldsymbol{s}}_{L}\right)$,

where $\left[\tilde{\boldsymbol{s}}_{q}\right]_{i}=1-\left[\boldsymbol{s}_{q}\right]_{i}$.

Given any evolutionary path $S$ and a symmetric path $\tilde{S}$ we can show that, if the antisymmetry-of-invasion property holds, the probabilities of each occurring are related in the following way:

$P(S)-P\left(S_{0}\right)=P\left(S_{0}\right)-P(\tilde{S})$,

at least to $\mathcal{O}(\delta)$. Here, $S_{0}$ is the evolutionary path with neutral selection $\delta=0$, i.e. all individuals have the same fitness. Thus, if any 


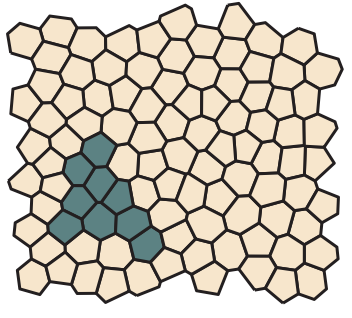

(a) Original state

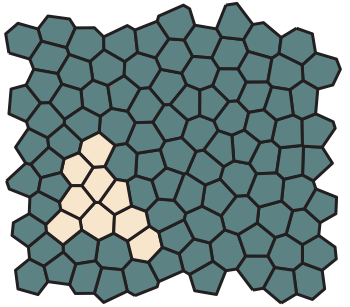

(b) Symmetric state.
Fig. 15. Symmetric states. (a) a mutant clone of $A$-players is invading a population of $B$-players. (b) a mutant clone of $B$-players is invading a population of $A$-players. If the antisymmetry-of-invasion property holds a given $A$-player in state (a) has payoff $a_{j}$, the equivalent $B$-player in state (b) has payoff $b_{k-j}=Q-a_{j}$.

given path has an advantage over the neutral process, the symmetric path must have an equivalent disadvantage.

We can further show that the following relation between the fixation probability for an $A$-player and the fixation probability of a $B$-player, denoted by $\rho_{A}$ and $\rho_{B}$, respectively, must hold:

$\rho_{A}-1 / Z=1 / Z-\rho_{B}$,

again to $\mathcal{O}(\delta)$. Recall that $\rho_{0}=1 / Z$ is the fixation probability for a neutral mutant. Thus, antisymmetry-of-invasion ensures that $\rho_{A}>\rho_{0}$ implies $\rho_{B}<\rho_{0}$, and hence that the conditions for $A$ or $B$ to be favourable are the same as to be beneficial.

Proof of Eq. 74. Consider a path $S$ as described by Eq. 72. The transition probability from state $\left(G_{q}, \boldsymbol{s}_{q}\right)$ to $\left(G_{q+1}, \boldsymbol{s}_{q+1}\right)$ is given by

$$
\begin{aligned}
P\left(\left(G_{q}, \boldsymbol{s}_{q}\right) \rightarrow\left(G_{q+1}, \boldsymbol{s}_{q+1}\right)\right) & =P\left(\boldsymbol{s}_{q} \rightarrow \boldsymbol{s}_{q+1}\right) \cdot P\left(G_{q} \rightarrow G_{q+1} \mid \boldsymbol{s}_{q} \rightarrow \boldsymbol{s}_{q+1}\right) \\
& =\frac{1}{Z^{2}}\left\{1+\delta\left[\pi_{\mathrm{birth}}\left(G_{q}, \boldsymbol{s}_{q}\right)-\pi\left(G_{q}, \boldsymbol{s}_{q}\right)\right]\right\} \cdot \psi_{q},
\end{aligned}
$$

where $\pi_{\text {birth }}$ is the payoff of the proliferating individual and $\pi$ is the average payoff in the population. The probabilities for transitions between graphs are given by $P\left(G_{q} \rightarrow G_{q+1} \mid \boldsymbol{s}_{q} \rightarrow \boldsymbol{s}_{q+1}\right)=\psi_{q}$.

The probability of $S$ occurring, given initial state $\left(G_{0}, \boldsymbol{s}_{0}\right)$, is given by multiplying the transition probabilities, i.e.

$P(S)=\prod_{q=0}^{L-1} P\left(\left(G_{q}, \boldsymbol{s}_{q}\right) \rightarrow\left(G_{q+1}, \boldsymbol{s}_{q+1}\right)\right)$,

which in the weak selection limit $\delta \rightarrow 0$ becomes

$P(S)=\frac{1}{Z^{2 L}}(1+\delta X(S)) \Psi(S)+\mathcal{O}\left(\delta^{2}\right)$.

Here,

$X(S)=\sum_{q=0}^{L-1}\left(\pi_{\text {birth }}\left(G_{q}, \boldsymbol{s}_{q}\right)-\pi\left(G_{q}, \boldsymbol{s}_{q}\right)\right)$

and

$\Psi(S)=\prod_{q=0}^{L-1} \psi_{q}$

The symmetric evolutionary path $\tilde{S}$ is equivalent to $S$, except that every individual has flipped its type. We assume that, in the weak selection limit at least, graph transitions do not depend on type, and thus, $\Psi(\tilde{S})=\Psi(S)$. The payoffs of course do depend on type, thus we write

$X(\tilde{S})=\sum_{q=0}^{L-1}\left(\pi_{\text {birth }}\left(G_{q}, \tilde{\boldsymbol{s}}_{q}\right)-\pi\left(G_{q}, \tilde{\boldsymbol{s}}_{q}\right)\right)$.
If the antisymmetry-of-invasion property, defined by Eq. 70, holds then

$X(\tilde{S})=\sum_{q=0}^{L-1}\left(\left(Q-\pi_{\text {birth }}\left(G_{q}, \boldsymbol{s}_{q}\right)\right)-\left(Q-\pi\left(G_{q}, \boldsymbol{s}_{q}\right)\right)\right)=-X(S)$.

Therefore, substituting into Eq. 78, we obtain

$P(\tilde{S})=\frac{1}{Z^{2 L}}(1-\delta X(S)) \Psi(S)+\mathcal{O}\left(\delta^{2}\right)$.

Setting $\delta=0$ gives $P\left(S_{0}\right)=\Psi(S) / Z^{2 L}$. Therefore, by summing Eqs. 78 and 83 , we obtain $P(S)+P(\tilde{S})=2 P\left(S_{0}\right)$ to $O(\delta)$, from which Eq. 74 follows.

Proof of Eq. 75. The fixation probability for a single initial $A$ player is obtained by summing $P\left(S_{i}\right)$ over all paths $S_{i}$ that start with a single initial $A$-player, and end with fixation for $A$-players. Summing over Eq. 78, we obtain

$$
\begin{aligned}
\rho_{A} & =\sum_{i} \frac{\Psi\left(S_{i}\right)}{Z^{2 L\left(S_{i}\right)}}+\sum_{i} \frac{\delta \Psi\left(S_{i}\right)}{Z^{2 L(}\left(S_{i}\right)} X\left(S_{i}\right)+\mathcal{O}\left(\delta^{2}\right) \\
& =\frac{1}{Z}+\sum_{i} \frac{\delta \Psi\left(S_{i}\right)}{Z^{2 L\left(S_{i}\right)}} X\left(S_{i}\right)+\mathcal{O}\left(\delta^{2}\right),
\end{aligned}
$$

where we have used the fact that the fixation probability for neutral selection $(\delta=0)$ is $\rho_{0}=1 / Z$. The fixation probability for $B$-players can similarly be obtained by summing $P\left(\tilde{S}_{i}\right)$ over all paths $\tilde{S}_{i}$ that start from a single $B$-player and end with $B$-player fixation. Thus,

$\rho_{B}=\frac{1}{Z}-\sum_{i} \frac{\delta \Psi\left(S_{i}\right)}{Z^{2 L\left(S_{i}\right)}} X\left(S_{i}\right)+\mathcal{O}\left(\delta^{2}\right)$.

Summing Eq. (84) and (85) gives us $\rho_{A}+\rho_{B}=2 / Z$ to $O(\delta)$, and thus Eq. 75.

\section{D.3. Antisymmetry-of-invasion for public goods games in epithelia}

In Section 3 we considered the conditions under which cooperative mutants are beneficial and favoured for sigmoid public goods games in the VT model with global updating and for the wellmixed population. Cooperation is beneficial when $b / c>(b / c)_{0}^{*}$ and favoured when $b / c>(b / c)_{1}^{*}$. It is is evident from Fig. 11 , that in general $(b / c)_{0}^{*} \neq(b / c)_{1}^{*}$. However, it appears in the figure that they are equal when $h=0.5$ and/or $s \rightarrow \infty$. Recall, that $h$ is the inflection point and $s$ is the steepness of the logistic function, defined by Eq. 5. Here, we show that both cases satisfy the antisymmetry-of-invasion property defined by Eq. 70 and thus $(b / c)_{0}^{*}=(b / c)_{1}^{*}$ must hold.

When $s \rightarrow 0$ we approach the NPD, which has a linear benefit function, given by Eq. 3. The cooperator and defector payoffs are thus

$a_{j, k}=b \cdot\left(\frac{j+1}{k+1}\right)-c \quad$ and $\quad b_{j, k}=b \cdot\left(\frac{j}{k+1}\right)$,

respectively. We therefore obtain

$a_{j, k}+b_{k-j, k}=b-c$.

As $b-c$ is a constant independent of $j$ and $k$, this satisfies antisymmetry-of-invasion, defined by Eq. 70 . The critical benefitto-cost ratio above which cooperation is favoured must, therefore, be equal to the critical benefit-to-cost ratio above which cooperation is beneficial, i.e. $(\boldsymbol{b} / \boldsymbol{c})_{0}^{*}=(\boldsymbol{b} / \boldsymbol{c})_{1}^{*}$.

We can also show that antisymmetry-of-invasion is satisfied when $h=0.5$. The sigmoid benefit function, defined by Eq. 4, has the symmetry property $\beta(x)=1-\beta(1-x)$ when $h=0.5$. The cooperator and defector payoffs are therefore given by 


$$
\begin{aligned}
a_{j, k} & =b \cdot \beta\left(\frac{j+1}{k+1}\right)-c=b \cdot\left[1-\beta\left(\frac{k-j}{k+1}\right)\right]-c \\
b_{j, k} & =b \cdot \beta\left(\frac{j}{k+1}\right),
\end{aligned}
$$

respectively. Once again, we find that $a_{j, k}+b_{k-j, k}=b-c$. Therefore, there is antisymmetry-of-invasion when $h=0.5$, so $(b / c)_{0}^{*}=(b / c)_{1}^{*}$ must hold.

\section{References}

Allen, B., Nowak, M.A., 2012. Evolutionary shift dynamics on a cycle. J. Theor. Biol. 311, 28-39. https://doi.org/10.1016/j.jtbi.2012.07.006. URL: https:// www.sciencedirect.com/science/article/pii/S0022519312003402?via\%3Dihub.

Allen, B., Lippner, G., Chen, Y.T., Fotouhi, B., Momeni, N., Yau, S.T., Nowak, M.A., 2016. Evolutionary dynamics on any population structure. Nature 544 (7649), 227-230. https://doi.org/10.1038/nature21723. arXiv:1605.06530.

Altrock, P.M., Liu, L.L., Michor, F., 2015. The mathematics of cancer: Integrating quantitative models. Nat. Rev. Cancer 15 (12), 730-745. https://doi.org/ 10.1038/nrc4029. URL: www.nature.com/reviews/cancer.

Antal, T. Ohtsuki, H., Wakeley, J., Taylor, P.D., Nowak, M.A., 2009. Evolution of cooperation by phenotypic similarity. Proc. Natl. Acad. Sci. U.S.A. 106 (21), 8597-8600. https://doi.org/10.1073/pnas.0902528106. URL: www.pnas.org/ cgi/content/full/.

Archetti, M., 2009a. The volunteer's dilemma and the optimal size of a social group. J. Theor. Biol. 261 (3), 475-480. https://doi.org/10.1016/j.jtbi.2009.08.018.

Archetti, M., 2009b. Cooperation as a volunteer's dilemma and the strategy of conflict in public goods games. J. Evol. Biol. 22 (11), 2192-2200. https://doi.org/ 10.1111/j.1420-9101.2009.01835.x.

Archetti, M., 2013a. Evolutionarily stable anti-cancer therapies by autologous cell defection. Evol. Med. Public Health 2013 (1), 161-172. https://doi.org/10.1093/ emph/eot014.

Archetti, M., 2013b. Evolutionary game theory of growth factor production: Implications for tumour heterogeneity and resistance to therapies. Br. J. Cancer 109 (4), 1056-1062. https://doi.org/10.1038/bjc.2013.336. URL: http://www.nature.com/articles/bjc2013336.

Archetti, M., 2013c. Dynamics of growth factor production in monolayers of cancer cells and evolution of resistance to anticancer therapies. Evol. Appl. 6 (8), 11461159. https://doi.org/10.1111/eva.12092.

Archetti, M., 2014. Evolutionary dynamics of the Warburg effect: glycolysis as a collective action problem among cancer cells. J. Theor. Biol. 341, 1-8. https:// doi.org/10.1016/j.jtbi.2013.09.017. URL: https:// www.sciencedirect.com/science/article/pii/S0022519313004451.

Archetti, M., 2016. Cooperation among cancer cells as public goods games on Voronoi networks. J. Theor. Biol. 396, 191-203. https://doi.org/10.1016/j. jtbi.2016.02.027. URL: https://www.sciencedirect.com/science/article/pii/ S0022519316001235.

Archetti, M., Pienta, K.J., 2019. Cooperation among cancer cells: applying game theory to cancer. Nat. Rev. Cancer 19 (2), 110-117. https://doi.org/10.1038/ s41568-018-0083-7. URL: www.nature.com/nrc.

Archetti, M., Scheuring, I., 2011. Coexistence of cooperation and defection in public goods games. Evolution 65 (4), 1140-1148. https://doi.org/10.1111/j.15585646.2010.01185.x.

Archetti, M., Scheuring, I., 2012. Review: Game theory of public goods in one-shot social dilemmas without assortment. J. Theor. Biol. 299, 9-20. https://doi.org/ 10.1016/j.jtbi.2011.06.018.

Archetti, M., Ferraro, D.A., Christofori, G., 2015. Heterogeneity for IGF-II production maintained by public goods dynamics in neuroendocrine pancreatic cancer. Proc. Natl. Acad. Sci. U.S.A. 112 (6), 1833-1838. https://doi.org/10.1073/ pnas.1414653112. URL: http://www.ncbi.nlm.nih.gov/pubmed/ 7506417http://www.pubmedcentral.nih.gov/articlerender.fcgi?artid= PMC42932.

Archetti, M., Scheuring, I., Yu, D.W., 2017. The non-tragedy of the non-linear commons, Preprints arXiv:2020040226, https://doi.org/10.20944/ preprints202004.0226.v1..

Axelrod, R., Axelrod, D.E., Pienta, K.J., 2006. Evolution of cooperation among tumor cells. Proc. Natl. Acad. Sci. U.S.A. 103 (36), 13474-13479. https://doi.org/ 10.1073/pnas.0606053103. URL: http://www.ncbi.nlm.nih.gov/pubmed/ 16938860, http://www.pubmedcentral.nih.gov/articlerender.fcgi?artid= PMC1557388.

Bach, L.A., Bentzen, S.M., Alsner, J., Christiansen, F.B., 2001. An evolutionary-game model of tumour-cell interactions: possible relevance to gene therapy. Eur. J. Cancer 37 (16), 2116-2120. https://doi.org/10.1016/S0959-8049(01)00246-5.

Bach, L.A., Sumpter, D.J., Alsner, J., Loeschcke, V., 2003. Spatial evolutionary games of interaction among generic cancer cells. J. Theor. Med. 5 (1), 47-58. https://doi. org/10.1080/10273660310001630443.

Bach, L.A., Helvik, T., Christiansen, F.B., 2006. The evolution of n-player cooperation - Threshold games and ESS bifurcations. J. Theor. Biol. 238 (2), 426-434. https:// doi.org/10.1016/j.jtbi.2005.06.007.

Basanta, D., Deutsch, A., 2008. A game theoretical perspective on the somatic evolution of cancer. In: Selected Topics in Cancer Modeling, Birkhäuser Boston. pp. 1-16. arXiv:0810.4738. doi:10.1007/978-0-8176-4713-1_5. URL: https:// arxiv.org/pdf/0810.4738.pdf..
Basanta, D., Hatzikirou, H., Deutsch, A., 2008b. Studying the emergence of invasiveness in tumours using game theory. Eur. Phys. J. B 63 (3), 393-397. https://doi.org/10.1140/epjb/e2008-00249-y. arXiv:0810.4724.

Basanta, D., Simon, M., Hatzikirou, H., Deutsch, A., 2008c. Evolutionary game theory elucidates the role of glycolysis in glioma progression and invasion. Cell Prolif. 41 (6), 980-987. https://doi.org/10.1111/j.1365-2184.2008.00563.x.

Basanta, D., Gatenby, R.A., Anderson, A.R., 2012. Exploiting evolution to treat drug resistance: Combination therapy and the double bind. Mol. Pharm. 9 (4), 914921. https://doi.org/10.1021/mp200458e. URL: https://pubs.acs.org/ sharingguidelines.

Cleary, A.S., Leonard, T.L., Gestl, S.A., Gunther, E.J., 2014. Tumour cell heterogeneity maintained by cooperating subclones in Wnt-driven mammary cancers. Nature 508 (1), 113-117. https://doi.org/10.1038/nature13187. URL: http:// www.nature.com/articles/nature13187.

Curtius, K., Wright, N.A., Graham, T.A., 2017. An evolutionary perspective on field cancerization. Nat. Rev. Cancer 18 (1), 19-32. https://doi.org/10.1038/ nrc.2017.102. URL: www.nature.com/nrc.

Eisenhoffer, G.T., Loftus, P.D., Yoshigi, M., Otsuna, H., Chien, C.B., Morcos, P.A., Rosenblatt, J., 2012. Crowding induces live cell extrusion to maintain homeostatic cell numbers in epithelia. Nature 484 (7395), 546-549. https:// doi.org/10.1038/nature10999.

Farhadifar, R., Röper, J.C., Aigouy, B., Eaton, S., Jülicher, F., 2007. The influence of cell mechanics, cell-cell interactions, and proliferation on epithelial packing. Curr. Biol. 17 (24), 2095-2104. https://doi.org/10.1016/j.cub.2007.11.049.

Fernandez-Gonzalez, R., Zallen, J.A., 2012. Feeling the squeeze: live-cell extrusion limits cell density in epithelia. Cell 149 (5), 965-967. https://doi.org/10.1016/ j.cell.2012.05.006.

Gatenby, R.A., Brown, J.S., 2020. Integrating evolutionary dynamics into cancer therapy. Nat. Rev. Clin. Oncol. 17 (11), 675-686. https://doi.org/10.1038/ s41571-020-0411-1. URL: www.nature.com/nrclinonc.

Gerlee, P., Altrock, P.M., 2017. Extinction rates in tumour public goods games. J. R. Soc. Interface 14 (134). https://doi.org/10.1098/rsif.2017.0342.

Gokhale, C.S., Traulsen, A., 2010. Evolutionary games in the multiverse. Proc. Natl. Acad. Sci. U.S.A. 107 (12), 5500-5504. https://doi.org/10.1073/ pnas.0912214107. arXiv:1003.5839.

Hanahan, D., Weinberg, R.A., 2000. The hallmarks of cancer. Cell 100, 57-70. https:// doi.org/10.1007/s00262-010-0968-0. arXiv:0208024, URL: http://www.ncbi. nlm.nih.gov/pubmed/10647931.

Hanahan, D., Weinberg, R.A., 2011. Hallmarks of cancer: the next generation. Cell 144 (5), 646-674. https://doi.org/10.1016/j.cell.2011.02.013. arXiv:0208024.

Hauert, C., De Monte, S., Hofbauer, J., Sigmund, K., 2002. Volunteering as Red Queen mechanism for cooperation in public goods games. Science 296 (5570), 11291132. https://doi.org/10.1126/science.1070582.

Hummert, S., Bohl, K., Basanta, D., Deutsch, A., Werner, S., Theißen, G., Schroeter, A., Schuster, S., 2014. Evolutionary game theory: Cells as players. Mol. BioSyst. 10 (12), 3044-3065. https://doi.org/10.1039/c3mb70602h. URL: http://xlink.rsc. org/?DOI=C3MB70602 H.

Inda, M.D.M., Bonavia, R., Mukasa, A., Narita, Y., Sah, D.W., Vandenberg, S., Brennan, C., Johns, T.G., Bachoo, R., Hadwiger, P., Tan, P., DePinho, R.A., Cavenee, W., Furnari, F., 2010. Tumor heterogeneity is an active process maintained by a mutant EGFR-induced cytokine circuit in glioblastoma. Genes Develop. 24 (16), 1731-1745. https://doi.org/10.1101/gad.1890510. URL: http://www.genesdev. org.

Jouanneau, J., Moens, G., Bourgeois, Y., Poupon, M.F., Thiery, J.P., 1994. A minority of carcinoma cells producing acidic fibroblast growth factor induces a community effect for tumor progression. Proc. Natl. Acad. Sci. U.S.A. 91 (1), 286-290. URL: http://www.ncbi.nlm.nih.gov/pubmed/7506417, http://www.pubmedcentral. nih.gov/articlerender.fcgi?artid=PMC42932.

Kaznatcheev, A., Vander Velde, R., Scott, J.G., Basanta, D., 2017. Cancer treatment scheduling and dynamic heterogeneity in social dilemmas of tumour acidity and vasculature. Br. J. Cancer 116 (6), 785-792. https://doi.org/10.1038/ bjc.2017.5. URL: www.bjcancer.com.

Kaznatcheev, A., Peacock, J., Basanta, D., Marusyk, A., Scott, J.G., 2019. Fibroblasts and alectinib switch the evolutionary games played by non-small cell lung cancer. Nat. Ecol. Evol. 3 (3), 450-456. https://doi.org/10.1038/s41559-0180768-z.

Lieberman, E., Hauert, C., Nowak, M.A., 2005. Evolutionary dynamics on graphs. Nature 433 (7023), 312-316. https://doi.org/10.1038/nature03204.

Maciejewski, W., Fu, F., Hauert, C., 2014. Evolutionary game dynamics in populations with heterogenous structures. PLoS Comput. Biol. 10, (4) . https:// doi.org/10.1371/journal.pcbi.1003567. arXiv:1312.2942 e1003567.

Marusyk, A., Tabassum, D.P., Altrock, P.M., Almendro, V., Michor, F., Polyak, K., 2014 Non-cell-autonomous driving of tumour growth supports sub-clonal heterogeneity. Nature. https://doi.org/10.1038/nature13556.

Masuda, N., 2009. Directionality of contact networks suppresses selection pressure in evolutionary dynamics. J. Theor. Biol. 258 (2), 323-334. https://doi.org/ 10.1016/j.jtbi.2009.01.025.

McClatchey, A.I., Yap, A.S., 2012. Contact inhibition (of proliferation) redux. Curr. Opin. Cell Biol. 24 (5), 685-694. https://doi.org/10.1016/j.ceb.2012.06.009.

Meineke, F.A., Potten, C.S., Loeffler, M., 2001. Cell migration and organization in the intestinal crypt using a lattice-free model. Cell Prolif. 34 (4), 253-266. https:// doi.org/10.1046/j.0960-7722.2001.00216.x. arXiv:0034902642.

Mesa, K.R., Kawaguchi, K., Cockburn, K., Gonzalez, D., Boucher, J., Xin, T., Klein, A.M. Greco, V., 2018. Homeostatic epidermal stem cell self-renewal is driven by local differentiation. Cell Stem Cell 23 (5), 677-686. https://doi.org/10.1016/j. stem.2018.09.005. 
Mirams, G.R., Fletcher, A.G., Maini, P.K., Byrne, H.M., 2012. A theoretical investigation of the effect of proliferation and adhesion on monoclonal conversion in the colonic crypt. J. Theor. Biol. 312, 143-156. https://doi.org/ 10.1016/j.jtbi.2012.08.002.

Moran, P.A., 1958. Random processes in genetics. Math. Proc. Cambridge Philos. Soc. 54 (1), 60-71. https://doi.org/10.1017/S0305004100033193. URL: http://www. journals.cambridge.org/abstract_S0305004100033193.

Nathanson, C.G., Tarnita, C.E., Nowak, M.A., 2009. Calculating evolutionary dynamics in structured populations. PLoS Comput. Biol. 5, (12) . https://doi. org/10.1371/journal.pcbi.1000615 e1000615.

Nowak, M.A., 2006. Five rules for the evolution of cooperation. Science 314 (5805), 1560-1563. https://doi.org/10.1126/science.1133755. URL: https://www.ncbi. nlm.nih.gov/pmc/articles/PMC3279745/pdf/nihms49939.pdf.

Nowak, M.A., Tarnita, C.E., Antal, T., 2010. Evolutionary dynamics in structured populations. Philos. Trans. R. Soc. B 365 (1537), 19-30. https://doi.org/10.1098/ rstb.2009.0215. URL: http://rstb.royalsocietypublishing.org/content/365/1537/ 19.short.

Ohtsuki, H., Hauert, C., Lieberman, E., Nowak, M.A., 2006a. A simple rule for the evolution of cooperation on graphs and social networks. Nature 441 (7092), 502-505. https://doi.org/10.1038/nature04605. arXiv:NIHMS150003.

Ohtsuki, H., Hauert, C., Lieberman, E., Nowak, M.A., 2006b. A simple rule for the evolution of cooperation on graphs and social networks. Nature 441 (7092), 502-505. https://doi.org/10.1038/nature04605. arXiv:NIHMS150003.

Osborne, J.M., Fletcher, A.G., Pitt-Francis, J.M., Maini, P.K., Gavaghan, D.J., 2017. Comparing individual-based approaches to modelling the self-organization of multicellular tissues. PLoS Comput. Biol. 13, (2) . https://doi.org/10.1371/ journal.pcbi.1005387 e1005387.

Pacheco, J.M., Santos, F.C., Souza, M.O., Skyrms, B., 2009. Evolutionary dynamics of collective action in N-person stag hunt dilemmas. Proc. R. Soc. B 276 (1655), 315-321. https://doi.org/10.1098/rspb.2008.1126.

Pavlogiannis, A., Chatterjee, K., Adlam, B., Nowak, M.A., 2015. Cellular cooperation with shift updating and repulsion. Scientific Rep. 5 (17147). https://doi.org/ 10.1038/srep17147. URL: http://www.nature.com/articles/srep17147.

Peña, J., Wu, B., Arranz, J., Traulsen, A., 2016. Evolutionary games of multiplayer cooperation on graphs. PLoS Comput. Biol. 12 (8), 1-15. https://doi.org/10.1371/ journal.pcbi.1005059.

Renton, J., Page, K.M., 2019. Evolution of cooperation in an epithelium. J. R. Soc. Interface 16 (152). https://doi.org/10.1098/rsif.2018.0918. 20180918.

Rockne, R.C., Hawkins-Daarud, A., Swanson, K.R., Sluka, J.P., Glazier, J.A., Macklin, P., Hormuth, D.A., Jarrett, A.M., Lima, E.A., Tinsley Oden, J., Biros, G., Yankeelov, T.E., Curtius, K., Al Bakir, I., Wodarz, D., Komarova, N., Aparicio, L., Bordyuh, M., Rabadan, R., Finley, S.D., Enderling, H., Caudell, J., Moros, E.G., Anderson, A.R., Gatenby, R.A., Kaznatcheev, A., Jeavons, P., Krishnan, N., Pelesko, J., Wadhwa, R. R., Yoon, N., Nichol, D., Marusyk, A., Hinczewski, M., Scott, J.G., 2019. The 2019 mathematical oncology roadmap. Phys. Biol. 16 (4), 41005. https://doi.org/ 10.1088/1478-3975/ab1a09.

Romijn, L.B., Almet, A.A., Tan, C.W., Osborne, J.M., 2020. Modelling the effect of subcellular mutations on the migration of cells in the colorectal crypt. BMC Bioinf. 21 (1). https://doi.org/10.1186/s12859-020-3391-3.

Santos, F.C., Santos, M.D., Pacheco, J.M., 2008. Social diversity promotes the emergence of cooperation in public goods games. Nature 454 (7201), 213216. https://doi.org/10.1038/nature06940. URL: www.nature.com/nature.

Souza, M.O., Pacheco, J.M., Santos, F.C., 2009. Evolution of cooperation under Nperson snowdrift games. J. Theor. Biol. 260 (4), 581-588. https://doi.org/ 10.1016/j.jtbi.2009.07.010.

Tarnita, C.E., Antal, T., Ohtsuki, H., Nowak, M.A., 2009a. Evolutionary dynamics in set structured populations. Proc. Natl. Acad. Sci. U.S.A. 106 (21), 8601-8604. https://doi.org/10.1073/pnas.0903019106. URL: http://www.ncbi.nlm.nih.gov/ pubmed/19433793, http://www.pubmedcentral.nih.gov/articlerender.fcgi? artid=PMC2689033
Tarnita, C.E., Ohtsuki, H., Antal, T., Fu, F., Nowak, M.A., 2009b. Strategy selection in structured populations. J. Theor. Biol. 259 (3), 570-581. https://doi.org/10.1016/ j.jtbi.2009.03.035.

Tomlinson, I.P.M., 1997. Game-theory models of interactions between tumour cells. Eur. J. Cancer Part A 33 (9), 1495-1500. https://doi.org/10.1016/S0959-8049 (97)00170-6.

Tomlinson, I.P.M., Bodmer, W.F., 1997. Modelling the consequences of interactions between tumour cells. Br. J. Cancer 75 (2), 157-160. https://doi.org/10.1038/ bjc.1997.26. URL: http://www.nature.com/articles/bjc199726.

Traulsen, A., Hauert, C., 2009. Stochastic Evolutionary Game Dynamics. In: H.G. Schuster (Ed.), Reviews of nonlinear dynamics and complexity, vol. 2, WileyVCH, Ch. 1, pp. 25-61. arXiv:0811.3538. doi:10.1016/B978-0-444-537669.00006-9. URL: http://web.evolbio.mpg.detraulsen/paper/05.pdf..

Van Leeuwen, I.M., Mirams, G.R., Walter, A., Fletcher, A., Murray, P., Osborne, J., Varma, S., Young, S.J., Cooper, J., Doyle, B., Pitt-Francis, J., Momtahan, L., Pathmanathan, P., Whiteley, J.P., Chapman, S.J., Gavaghan, D.J., Jensen, O.E., King, J.R., Maini, P.K., Waters, S.L., Byrne, H.M., 2009. An integrative computational model for intestinal tissue renewal. Cell Prolif. 42 (5), 617-636. https://doi.org/ 10.1111/j.1365-2184.2009.00627.x. arXiv:69549138171.

Waclaw, B., Bozic, I., Pittman, M.E., Hruban, R.H., Vogelstein, B., Nowak, M.A., 2015. A spatial model predicts that dispersal and cell turnover limit intratumour heterogeneity. Nature 525 (7568), 261-264. https://doi.org/ 10.1038 /nature 14971.

Warburg, O., 1956. On the origin of cancer cells. Science 123 (3191), 309-314. https://doi.org/10.1126/science.123.3191.309. arXiv:NIHMS150003. URL: http://www.jstor.org/stable/1750066\%5Cn, http://www.jstor.org/stable/ 1750066 ? seq=1\&cid=pdf-reference\#references_tab_contents\%5Cn, http:// about.jstor.org/terms.

West, J., Ma, Y., Newton, P.K., 2018. Capitalizing on competition: an evolutionary model of competitive release in metastatic castration resistant prostate cancer treatment. J. Theor. Biol. 455, 249-260. https://doi.org/10.1016/j. jtbi.2018.07.028.

West, J., Schenck, R.O., Gatenbee, C., Robertson-Tessi, M., Anderson, A.R.A., 2021. Normal tissue architecture determines the evolutionary course of cancer. Nat. Commun. 12 (1), 1-9. https://doi.org/10.1038/s41467-021-22123-1.

Wölfl, B., te Rietmole, H., Salvioli, M., Thuijsman, F., Brown, J.S., Burgering, B., Stanková, K., 2020. The contribution of evolutionary game theory to understanding and treating cancer. medRxiv. https://doi.org/10.1101/ 2020.12.02.20241703. arXiv:2020.12.02.20241703.

Wu, B., Traulsen, A. Gokhale, C.S., 2013. Dynamic properties of evolutionary multiplayer games in finite populations. Games 4 (2), 182-199. https://doi.org/ 10.3390/g4020182. URL: http://www.journals.cambridge.org/abstract_ S0305004100033193.

You, L., Brown, J.S., Thuijsman, F., Cunningham, J.J., Gatenby, R.A., Zhang, J., Staková K., 2017. Spatial vs. non-spatial eco-evolutionary dynamics in a tumor growth model. Journal of Theoretical Biology 435, 78-97. https://doi.org/10.1016/j. jtbi.2017.08.022. URL: https://linkinghub.elsevier.com/retrieve/pii/ S0022519317303971.

Zhang, J., Cunningham, J.J., Brown, J.S., Gatenby, R.A., 2009. Integrating evolutionary dynamics into treatment of metastatic castrate-resistant prostate cancer. Nat. Commun. 8 (1). https://doi.org/10.1038/s41467-017-01968-5s. URL: www.nature.com/naturecommunications.

Zhou, H., Neelakantan, D., Ford, H.L., 2017. Clonal cooperativity in heterogenous cancers. Semin. Cell Develop. Biol. 64, 79-89. https://doi.org/10.1016/j. semcdb.2016.08.028.

Zukewich, J., Kurella, V., Doebeli, M., Hauert, C., 2013. Consolidating birth-death and death-birth processes in structured populations. PLoS ONE 8, (1). https://doi. org/10.1371/journal.pone.0054639. URL: www.plosone.org e54639. 